\title{
Utilisation de la modélisation de la qualité de l'eau en rivière pour l'évaluation du respect des normes en contexte de données restreintes : cas d'application du bassin versant de la rivière $\mathrm{Cau}$ \\ Modeling river water quality to assess standard compliance with restricted data: Case study of the Cau River watershed
}

\author{
Phu Duc Nguyen, Jean-Pierre Villeneuve, Babacar Toumbou, Sophie Duchesne \\ et Nomessi Kokutse
}

Volume 31, numéro 3, 2018

Reçu le 3 avril 2017, accepté le 9 janvier 2018

URI : https://id.erudit.org/iderudit/1054307ar

DOI : https://doi.org/10.7202/1054307ar

Aller au sommaire du numéro

Éditeur(s)

Université du Québec - INRS-Eau, Terre et Environnement (INRS-ETE)

ISSN

1718-8598 (numérique)

Découvrir la revue

Citer cet article

Nguyen, P., Villeneuve, J.-P., Toumbou, B., Duchesne, S. \& Kokutse, N. (2018). Utilisation de la modélisation de la qualité de l'eau en rivière pour l'évaluation du respect des normes en contexte de données restreintes : cas d'application du bassin versant de la rivière Cau. Revue des sciences de l'eau / Journal of Water Science, 31(3), 271-290. https://doi.org/10.7202/1054307ar
Résumé de l'article

La dégradation de la qualité de l'eau des bassins versants est principalement due au développement socio-économique, dont découlent la croissance démographique, l'urbanisation, l'industrialisation et l'intensification de l'agriculture. La gestion intégrée des ressources en eau par bassin versant (GIEBV) peut être appliquée pour limiter cet impact négatif. Le modèle GIBSI (Gestion Intégrée par Bassin versant à l'aide d’un Système Informatisé) est un outil qui peut être utilisé pour cette GIEBV. GIBSI inclut notamment un sous-modèle pour la production et le transport de l'azote et du phosphore (PO) ainsi qu'un sous-modèle pour la qualité de l'eau en rivière (Qual-GIBSI). Cette étude est axée principalement sur l'applicabilité de ces deux sous-modèles dans une zone tropicale avec un climat de type mousson dans un contexte de données restreintes. Cet exercice a comme objectif, entre autres, de valider si ces sous-modèles sont applicables dans ces conditions. L'application permet également de vérifier dans quelle mesure les normes de qualité de l'eau en rivière sont respectées. Le bassin versant de la rivière Cau au Vietnam est utilisé comme cas d'application. Nous avons démontré à travers cette étude que, même sur ce bassin versant tropical soumis à la mousson où les données disponibles sont limitées en quantité et en qualité, les modèles PO et Qual-GIBSI peuvent apporter des informations utiles pour la gestion de la qualité de l'eau. 


\section{UTILISATION DE LA MODÉLISATION DE LA QUALITÉ DE L'EAU EN RIVIÈRE POUR L'ÉVALUATION DU RESPECT DES NORMES EN CONTEXTE DE DONNÉES RESTREINTES : CAS D’APPLICATION DU BASSIN VERSANT DE LA RIVIÈRE CAU}

Modeling river water quality to assess standard compliance with restricted data: Case study of the Can River watershed

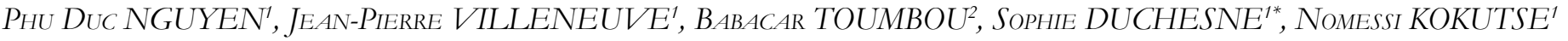

${ }^{1}$ Centre Eau Terre Environnement, Institut national de la recherche scientifique (INRS), 490, rue de la Couronne, Québec (Québec) G1K 9A9, Canada

${ }^{2}$ UFR Sciences Économiques et Sociales, Université de Thiès, BP 400, Thiès, Sénégal

Reçu le 3 avril 2017, accepté le 9 janvier 2018

\section{RÉSUMÉ}

La dégradation de la qualité de l'eau des bassins versants est principalement due au développement socio-économique, dont découlent la croissance démographique, l'urbanisation, l'industrialisation et l'intensification de l'agriculture. La gestion intégrée des ressources en eau par bassin versant (GIEBV) peut être appliquée pour limiter cet impact négatif. Le modèle GIBSI (Gestion Intégrée par Bassin versant à l'aide d'un Système Informatisé) est un outil qui peut être utilisé pour cette GIEBV. GIBSI inclut notamment un sous-modèle pour la production et le transport de l'azote et du phosphore (PO) ainsi qu'un sous-modèle pour la qualité de l'eau en rivière (Qual-GIBSI). Cette étude est axée principalement sur l'applicabilité de ces deux sous-modèles dans une zone tropicale avec un climat de type mousson dans un contexte de données restreintes. Cet exercice a comme objectif, entre autres, de valider si ces sous-modèles sont applicables dans ces conditions. L'application permet également de vérifier dans quelle mesure les normes de qualité de l'eau en rivière sont respectées. Le bassin versant de la rivière Cau au Vietnam est utilisé comme cas d'application. Nous avons démontré à travers cette étude que, même sur ce bassin versant tropical soumis à la mousson où les données disponibles sont limitées en quantité et en qualité, les modèles PO et Qual-GIBSI peuvent apporter des informations utiles pour la gestion de la qualité de l'eau.

Mots-clés : modélisation, zone tropicale, données rares, qualité de l'eau, bassin versant.

\begin{abstract}
The degradation of water quality in watersheds is mainly due to socio-economic development, including population
\end{abstract}


growth, urbanization, industrialization and intensification of agriculture. Integrated watershed resources management (IWRM) can be applied to limit this negative impact. The GIBSI model is a tool that can be used to apply IWRM. It includes a sub-model for nitrogen and phosphorus production and transport (PO), and a sub-model for river water quality (Qual-GIBSI). This study focuses mainly on the applicability of these two sub-models in a tropical zone with a monsoontype climate in a context of restricted data. This exercise will, among other things, validate whether these sub-models are applicable under these conditions. The application will also verify to what extent the river water quality standards are met. The Cau River watershed in Vietnam is used as a case study. We demonstrated through this study that even in this monsoon tropical catchment area where available data are limited in quantity and quality, the PO and Qual-GIBSI models can provide useful information for managing water quality.

Key words: mathematical model, tropical area, sparse data, water quality, watershed.

\section{INTRODUCTION}

L'eau douce ne représente qu'environ 2,5\% des ressources disponibles en eau dans le monde (SHIKLOMANOV, 1998). Une partie importante de cette quantité d'eau douce est difficile d'accès, car elle est constituée de réserves très profondes et de glace. Au total, moins de $1 \%$ des réserves planétaires est à la disposition de l'humanité. Outre une très mauvaise répartition des stocks, la croissance démographique et l'expansion économique constituent de lourdes menaces pour la quantité et la qualité de l'eau dans certains pays.

La gestion des ressources en eau s'appuie sur deux aspects, l'un quantitatif (gestion des stocks d'eau disponible) et l'autre qualitatif (pollution des ressources). Chacun de ces deux aspects est influencé par deux composantes : les composantes naturelles et les activités humaines. La première composante concerne la quantité et la qualité de l'eau douce disponible (p. ex. prévision des cycles hydrologiques) et sa mobilité dans le milieu terrestre (hydraulique, hydrogéologie, écoulements souterrains, échanges sol-atmosphère, ruissellement de surface, etc.). Le second aspect concerne de multiples contraintes que subit la gestion des ressources en eau comme les contraintes techniques (p. ex. infrastructures de transports d'eau), économiques (influence des activités humaines sur la qualité de la ressource), sociales et politiques (p. ex. les lois), entre autres.

Une gestion efficace des ressources en eau passe par la prise en compte de plusieurs volets : les activités économiques qui contribuent à la dégradation de la qualité de l'eau, l'aspect social en relation avec le développement des agglomérations et les rejets des eaux usées d'origine domestique (avec ou sans traitement), les rejets industriels, etc.

Ainsi, le premier objectif principal de cette étude est de modéliser la qualité de l'eau en rivière dans un contexte de données rares et de climat tropical de type mousson. Pour ce faire, deux sous-objectifs peuvent être identifiés : le premier est d'adapter les modèles de simulation de la qualité de l'eau sur le bassin versant et en rivière dans GIBSI aux conditions particulières des bassins versants soumis aux contraintes de données limitées ou rares et de climat tropical de type mousson; le second est de trouver une méthode appropriée pour le calage des modèles. Le second objectif principal de cet article est de démontrer comment les résultats des modèles de qualité de l'eau précédemment calés permettent de vérifier dans quelle mesure les normes de qualité de l'eau en rivière sont respectées, même dans les bassins versants où les données mesurées sont rares.

\section{REVUE DE LITTÉRATURE}

La modélisation de la qualité de l'eau est un sujet d'importance. Il existe plusieurs modèles développés dans le cadre de différentes études dans le monde portant sur ce sujet. Il s'agit, par exemple, des modèles Agricultural Non-Point Source pollution (AgNPS) (YOUNG et al., 1987), Qual2E (BROWN et BARNWELL, 1987), MIKE SHE (REFSGAARD et HANSEN, 1982), Soil and Water Assessment Tool (SWAT) (NEITSCH et al., 2005), ou Gestion Intégrée par Bassin versant à l'aide d'un Système Informatisé (GIBSI) (VILLENEUVE et al., 1998).

Les sources de pollution ponctuelle et diffuse contribuent à la dégradation de la qualité de l'eau en rivière d'un bassin versant. L'étude de l'impact de la pollution diffuse a commencé en Amérique du Nord, vers les années 1970, avec le programme d'identification et de contrôle des sources de pollution ponctuelle et diffuse du bassin versant des Grands Lacs du Canada et des États-Unis (ONGLEY, 1996). En 1972, les deux gouvernements du Canada et des États-Unis ont établi le Pollution from Land Use Activities Reference Group (PLUARG, 1979) pour étudier des sources de pollution du bassin versant des Grands Lacs. Cette étude, PLUARG, qui a été faite essentiellement dans les années 1970 et début 1980, a entraîné une amélioration considérable de la compréhension scientifique des impacts des activités d'utilisation du sol sur la qualité de l'eau. Ainsi, plusieurs outils et modèles ont été développés pour évaluer l'entraînement et le transport des sédiments, des nutriments et des pesticides dans beaucoup de domaines parmi lesquels l'hydrologie, l'érosion et le transport 
des polluants. Certains modèles comme Areal Nonpoint Source Watershed Environmental Response Simulation (ANSWERS) (BEASLEY et HUGGINS, 1981), AgNPS (YOUNG et al., 1987), Groundwater Loading Effects of Agricultural Management Systems (GLEAMS) (LEONARD et al. 1987; KNISEL, 1980) et SWAT (NEITSCH et al., 2011) ont été développés puis utilisés. De plus, un outil de gestion intégrée par bassin versant, GIBSI, a été développé au Québec (VILLENEUVE et al., 1998). Il a été conçu et développé au Centre Eau Terre Environnement de l'Institut national de la recherche scientifique (Québec, Canada). C'est un outil permettant de simuler des processus hydrologiques, de production et de transport de sédiments et de contaminants sur la surface du sol et aussi de simuler la qualité de l'eau en rivière.

\section{MATÉRIEL ET MÉTHODES}

\subsection{Matériel : outil utilisé (GIBSI)}

\subsubsection{Structure et fonctionnement}

GIBSI est un outil informatique qui permet aux gestionnaires del'eau d'explorer divers scénarios de gestion à l'échelle du bassin versant (ROUSSEAU et al., 2000). Tel qu'illustré à la figure 1, cet outil informatique d'aide à la gestion comprend une base de données (spatiales et attributs), un système d'information géographique (SIG), une base de données relationnelle (BD) et des modèles mécanistes de simulation (hydrologie, érosion des sols et en rivières, transport et transformation de l'azote, du phosphore et des pesticides dans les sols et qualité de l'eau dans les rivières et les lacs). GIBSI permet aux gestionnaires d'estimer quantitativement l'impact sur la qualité de l'eau de différents scénarios d'aménagement du territoire.

Ensuite, les simulations à l'aide des modèles permettent de déterminer l'effet des modifications apportées sur le régime hydrologique et la qualité de l'eau en tout point du réseau hydrographique.

Enfin, les outils d'analyse des résultats permettent d'analyser les résultats de simulations : graphiques, cartes, calcul de probabilités de dépassement et analyse avantage/coût.

\subsubsection{Modèles de simulation de GIBSI utilisés}

3.1.2.1 Modèle de transport des polluants PO

La modélisation des polluants en milieu terrestre est basée, dans GIBSI, sur les algorithmes du modèle de bassin versant SWAT (NEITSCH et al., 2011) et du modèle de productivité des sols EPIC (SHARPLEY et WILLIAMS, 1990). La modélisation des cycles de l'azote, du phosphore et des pesticides est réalisée par le modèle POPES. Le sousmodèle PO simule le cycle de l'azote et du phosphore alors que le sous-modèle PES simule le transport et la dégradation des pesticides. Le transport dépend des masses de sédiments provenant de l'érosion hydrique des sols et des volumes d'eau en mouvement à la surface et dans les couches de sol des diverses occupations agricoles (p. ex. maïs, pâturage, céréales) d'une unité hydrologique relativement homogène (UHRH). Une UHRH est définie comme un très petit sousbassin versant associé à un tronçon de rivière. L'objectif est d'effectuer les simulations sur ces unités hydrologiques, qui sont relativement homogènes par rapport au bassin étudié, et de conserver la structure interne d'écoulement de cette unité. Les formes d'azote et de phosphore sortant de l'UHRH sont respectivement l'azote minéral (nitrates), l'azote organique, le phosphore minéral labile et le phosphore organique. L'azote minéral et le phosphore minéral labile sont transportés par l'eau de ruissellement (et dans le cas de l'azote, également par l'eau de l'écoulement latéral du sol), tandis que l'azote organique et le phosphore organique sont transportés par les sédiments. Les pesticides dissous demeurent disponibles pour le transport par ruissellement, par écoulement vertical et par écoulement latéral, tandis que les pesticides adsorbés sont disponibles pour le transport par les sédiments.

\subsubsection{Modèle de qualité de l'eau en rivière Qual-GIBSI}

Le modèle de qualité retenu pour l'intégration au sein du système GIBSI est le modèle Qual2E (BROWN et BARNWELL, 1987). En outre, une modification significative du code original de ce modèle a été opérée pour l'adapter à GIBSI (VILLENEUVE et al., 1998), ce qui donne le modèle Qual-GIBSI. Plusieurs processus sont simulés par le modèle Qual-GIBSI. Ces processus sont regroupés selon quatre modules distincts, à savoir les modules de :

- transport-dispersion-dilution des concentrations;

- simulation des processus chimiques et biologiques locaux;

- température;

- érosion et transport des sédiments.

Les différentes variables simulées sur chaque tronçon $\mathrm{du}$ réseau hydrographique sont les suivantes : algues (chlorophylle $a$ ), azote organique $\left(\mathrm{N}_{\text {org }}\right)$, ammoniac $\left(\mathrm{N}_{-} \mathrm{NH}_{3}\right)$, nitrites $\left(\mathrm{N}-\mathrm{NO}_{2}\right)$, nitrates $\left(\mathrm{N}-\mathrm{NO}_{3}\right)$, demande en oxygène des sédiments $(\mathrm{DO})$, demande biologique en oxygène à cinq jours $\left(\mathrm{DBO}_{5}\right)$, phosphore organique $\left(\mathrm{P}_{\text {org }}\right)$, phosphore dissous $\left(\mathrm{P}_{\text {diss }}\right)$, oxygène dissous $(\mathrm{OD})$ et coliformes fécaux.

\subsection{Méthodes}

Étant donné que le logiciel GIBSI a été développé et appliqué au Québec, dans un contexte de disponibilité de données relativement abondantes, il s'avérait nécessaire de 


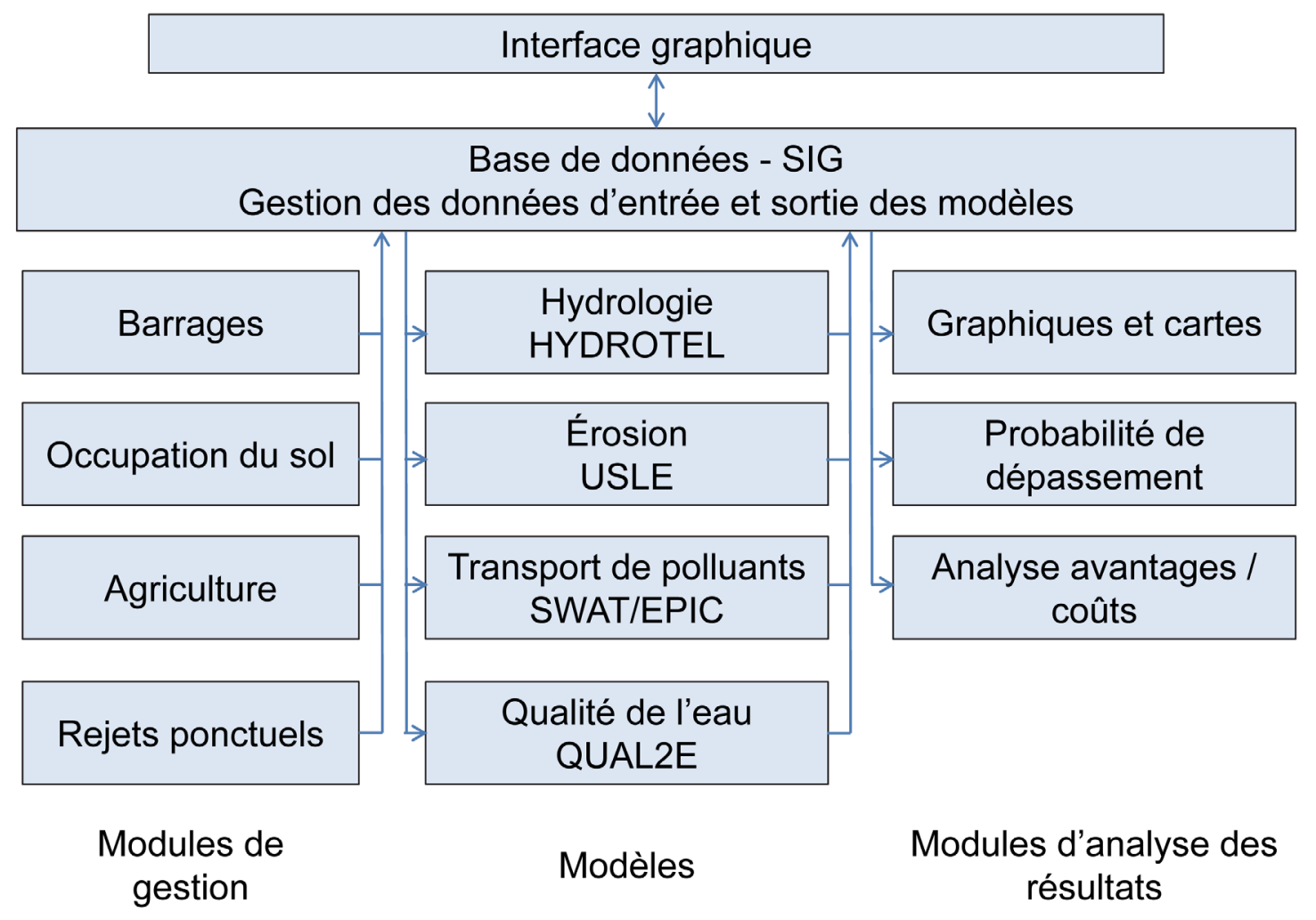

Figure 1. Représentation schématique du fonctionnement général de GIBSI (adaptée de QUILBÉ et ROUSSEAU, 2007).

Schematic representation of the general structure of GIBSI (adapted from QUILBÉ and ROUSSEAU, 2007).

l'adapter aux conditions des zones tropicales de type mousson et de telle sorte qu'il soit utilisable avec peu de données.

Quelques études ont été faites pour adapter certains sousmodèles de GIBSI aux conditions du bassin versant de la rivière Cau du Vietnam. Il s'agit des sous-modèles Hydrotel (qui modélise l'hydrologie du bassin versant; FORTIN et al., 1995) et MODEROSS (qui modélise l'érosion des sols; DUCHEMIN et al., 2001). Les sous-modèles Hydrotel et MODEROSS ont été adaptés au contexte du bassin versant de la rivière Cau par NGUYEN (2012) et PHAM (2013), respectivement. En ce qui concerne cette étude, les sous-modèles PO (qui modélise le transport de l'azote et du phosphore; NASR, 1996) et QualGIBSI (qui simule le transport, la dispersion et la dilution des concentrations ainsi que les transformations biochimiques; VILLENEUVE et al., 1998) seront adaptés aux conditions du bassin versant de la rivière Cau du Vietnam.

Une fois le logiciel GIBSI adapté aux conditions du bassin versant de la rivière Cau, l'approche utilisée pour modéliser la qualité de l'eau consiste en la simulation, dans un premier temps, du transport de l'azote et du phosphore en surface avec le modèle $\mathrm{PO}$, puis, dans un deuxième temps, de la qualité de l'eau en rivière en utilisant les résultats du modèle PO (quantités d'azote et de phosphore sortant du sol) comme sources de pollution diffuse dans le modèle Qual-GIBSI. Les sources pollution ponctuelles, entrées par l'utilisateur, et les résultats du sous-modèle hydrologique Hydrotel sont également prises en compte dans ce processus.

\subsubsection{Modélisation de la pollution diffuse avec PO}

Le modèle PO permet de modéliser les cycles de l'azote et du phosphore. Tel que mentionné précédemment, le transport dépend des masses de sédiments provenant de l'érosion hydrique des sols et des volumes d'eau en mouvement à la surface et dans les couches de sol des diverses occupations agricoles. Les deux formes d'azote et de phosphore sortant de l'UHRH pour chaque jour de simulation sont respectivement l'azote minéral (sous forme d'azote-nitrates), l'azote organique, le phosphore minéral labile et le phosphore organique.

\subsubsection{Cycle de l'azote}

L'azote dans le sol se présente sous deux formes : azote organique et azote minéral. L'azote organique est la forme prédominante (environ $95 \%$ de l'azote du sol). Le cycle l'azote tel que représenté dans $\mathrm{PO}$ est illustré à la figure 2 .

Le modèle $\mathrm{PO}$ ne considère que les nitrates comme azote minéral (sous la forme $\mathrm{NO}_{3}^{-}$). Cette simplification est due au fait que la minéralisation se produit assez rapidement dans les 


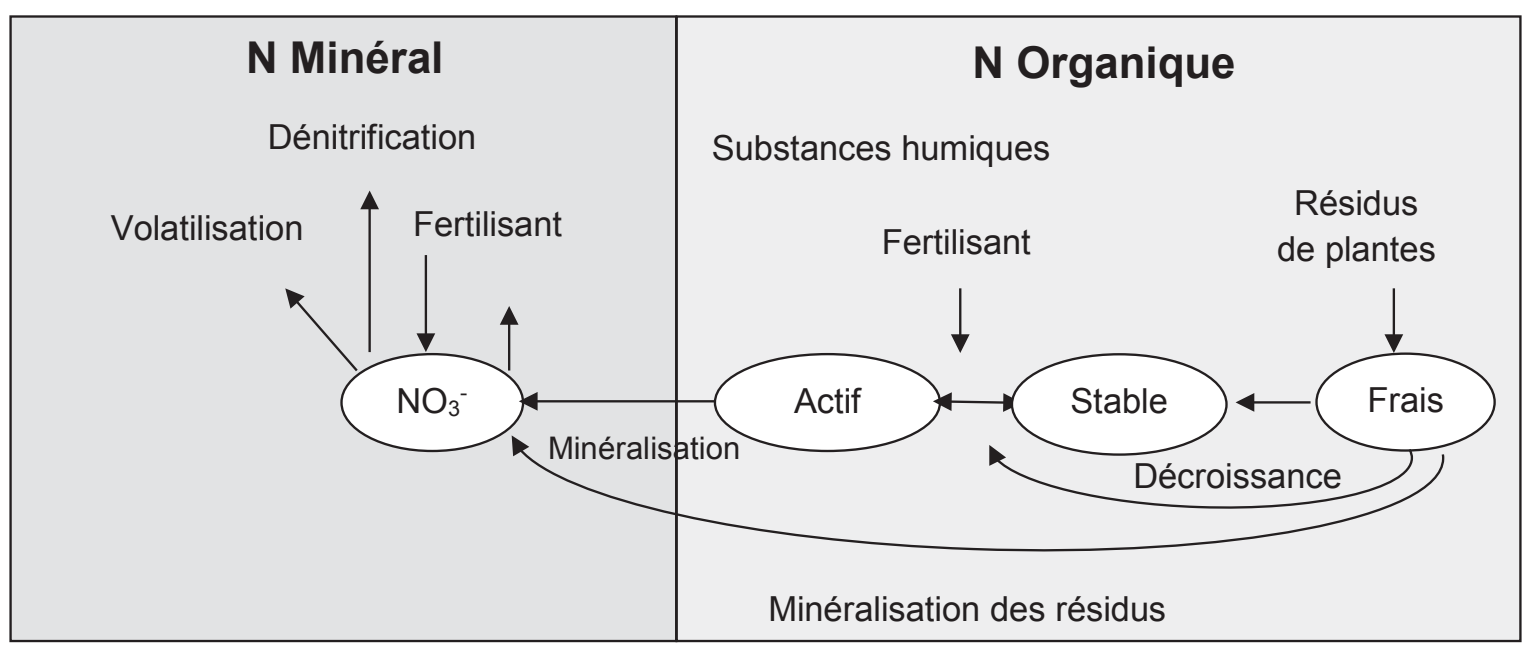

Figure 2. Cycle de l'azote.

Nitrogen cycle.

sols cultivés. On suppose qu’il y a seulement les nitrates qui contribuent à la croissance des plantes ou qui sont transportés par les eaux de l'écoulement vertical, du ruissellement et de l'écoulement latéral. En condition anaérobie de sol saturé, le processus de dénitrification contribue aux pertes en azote. Les nitrates peuvent également être immobilisés par les microorganismes du sol, qui eux contiennent une partie de l'azote sous forme organique (azote organique frais). En effet, l'azote organique est séparé en deux catégories : l'azote organique de l'humus et l'azote organique des résidus de culture (azote organique frais). L'azote organique de l'humus peut être plus ou moins adsorbé par les particules du sol. Ainsi, on y distingue deux catégories : l'azote organique stable (fortement adsorbé) et l'azote organique actif (légèrement adsorbé et disponible pour le processus de minéralisation). Quant à l'azote organique frais, il est en grande partie minéralisé. Pour ce qui est du transport de l'azote par les sédiments, le réservoir considéré est celui de l'azote organique de l'humus (azote organique stable et azote organique actif). La volatilisation de l'azote est aussi considérée lors de l'épandage des engrais (Figure 3).

POPES se base sur différentes équations pour décrire les processus de transport des nutriments ou les transformations entre les différentes composantes. Il fournit les quantités d'azote exportées à la rivière par les UHRH, ce qui comprend les charges exportées par les sédiments, par le ruissellement et par le débit de base (souterrain). Les équations du modèle, qui décrivent les phénomènes physiques relatifs à chaque UHRH, sont données dans VILLENEUVE et al. (1998).

\subsubsection{Cycle du phosphore}

Le phosphore organique est divisé en deux catégories : le phosphore organique des résidus de culture et le phosphore organique actif de l'humus. Pour ce qui est du phosphore minéral, il est réparti en trois groupes : labile, actif et stable.
Les échanges entre ces derniers groupes se font par processus d'adsorption et de désorption. Le phosphore minéral labile, bien que légèrement adsorbé, peut se désorber facilement. Il ne se retrouve donc jamais libre dans le sol. Ainsi, c'est le phosphore minéral labile qui est considéré pour le transport par les eaux de ruissellement. Pendant ce temps, le phosphore organique de l'humus et des résidus de culture se transforme en phosphore minéral par la minéralisation et l'inverse par le processus d'immobilisation pour le phosphore organique des résidus de culture. Quant au transport du phosphore sous forme particulaire, il est régi par le transport des sédiments et il se retrouve à la fois sous sa forme organique (phosphore organique de l'humus) et sous sa forme minérale (phosphore minéral actif et phosphore minéral stable), puisque ces formes sont toutes les deux adsorbées par les particules de sol. Le phosphore organique est transporté par les sédiments et le phosphore minéral labile par l'eau (Figure 4).

On note qu'il n'y a pas de phosphore dans les écoulements verticaux et latéraux de l'eau dans PO. Le phosphore est en général fortement adsorbé par les particules de sol et, par conséquent, est distribué dans la couche supérieure du sol. Lors des fertilisations organiques ou minérales, les deux catégories concernées sont le phosphore organique actif de l'humus et le phosphore minéral labile. Les sols cultivables, lorsque labourés, contribuent également à la réserve de phosphore organique frais des résidus de culture (Figure 5). Les équations du modèle sont fournies en détail dans VILLENEUVE et al. (1998).

\subsubsection{Modélisation de la qualité de l'eau en rivière avec Qual-GIBSI}

Quatre types de processus sont simulés par le modèle Qual-GIBSI. Ces processus sont regroupés selon quatre modules : a) transport-dispersion-dilution des concentrations, b) simulation des processus chimiques et biologiques locaux, 


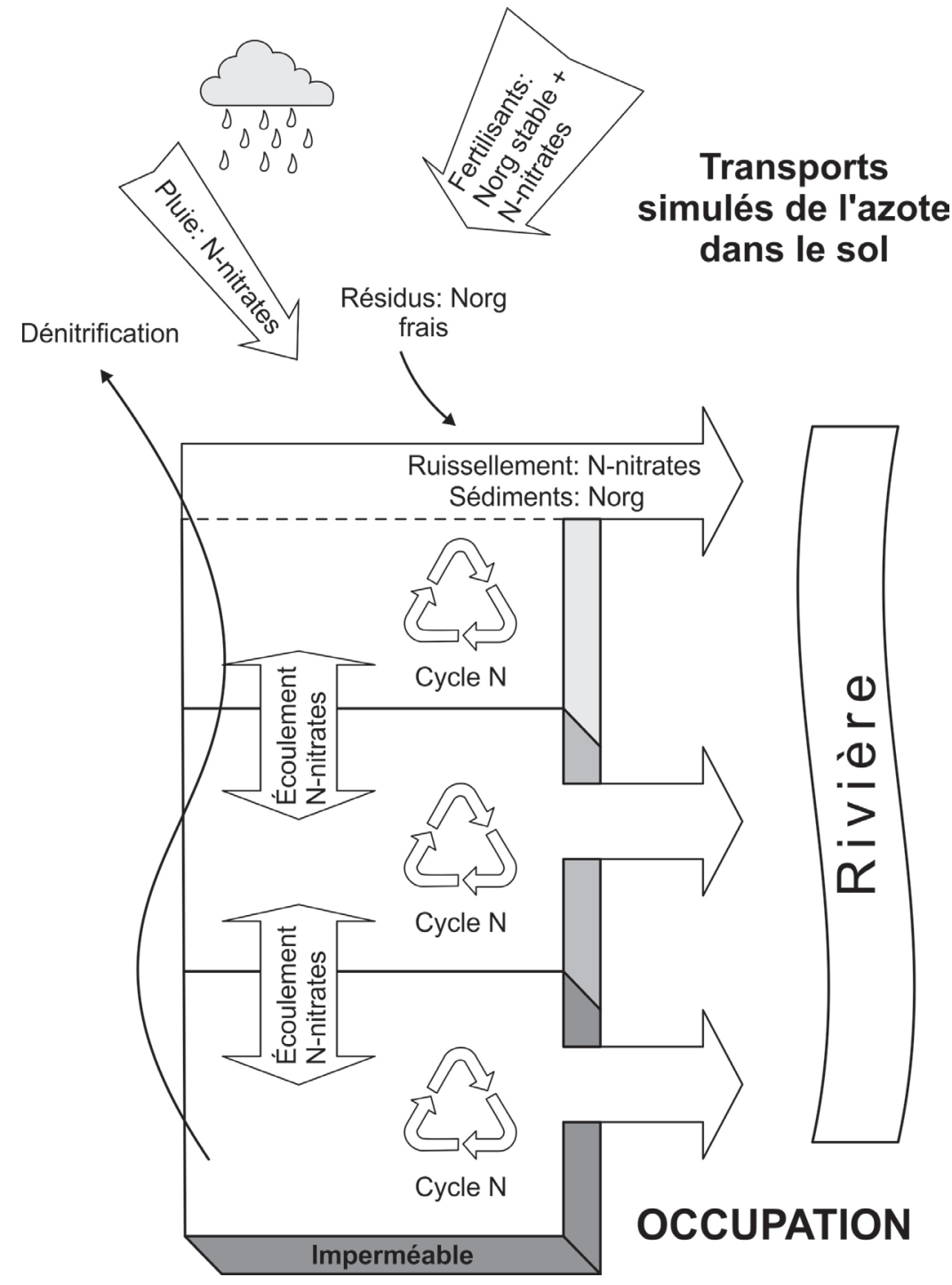

Figure 3. Transport simulé de l'azote dans le sol (VILLENEUVE et al., 1998). Simulated transport of nitrogen in soil (VILLENEUVE et al., 1998).

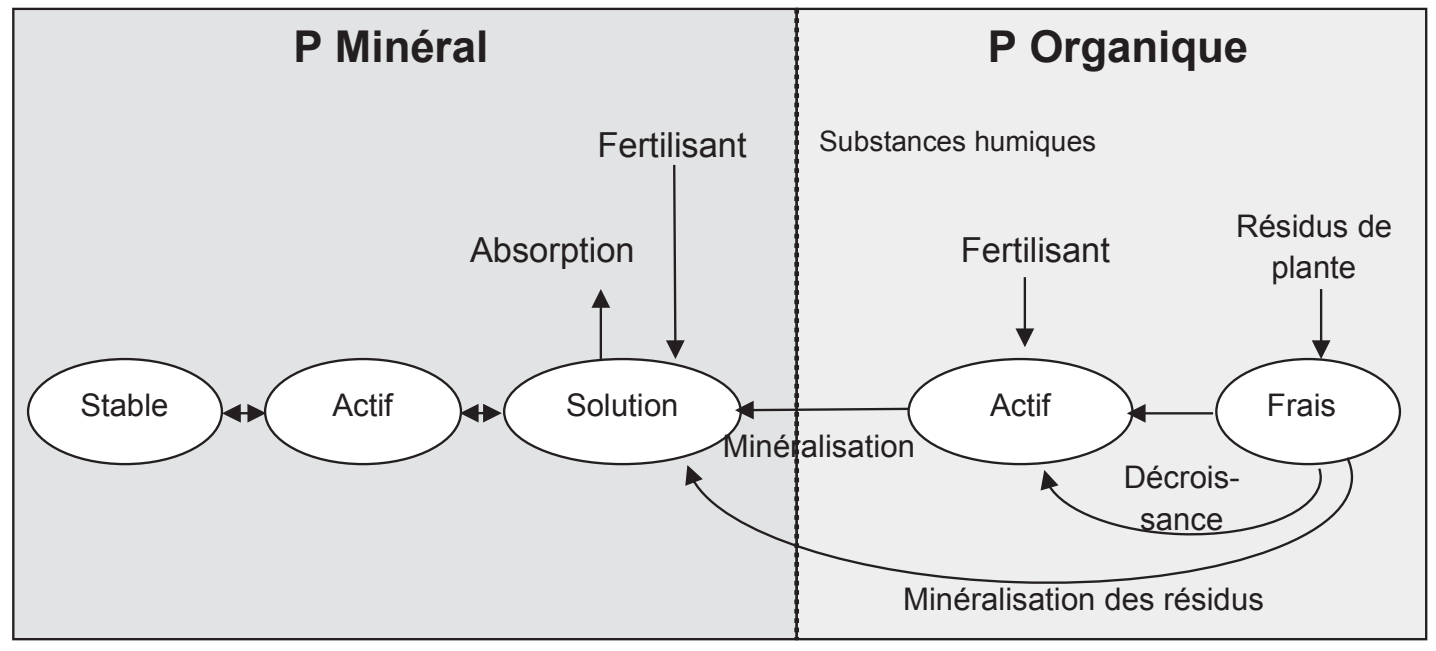

Figure 4. Cycle du phosphore.

Phosphorus cycle. 


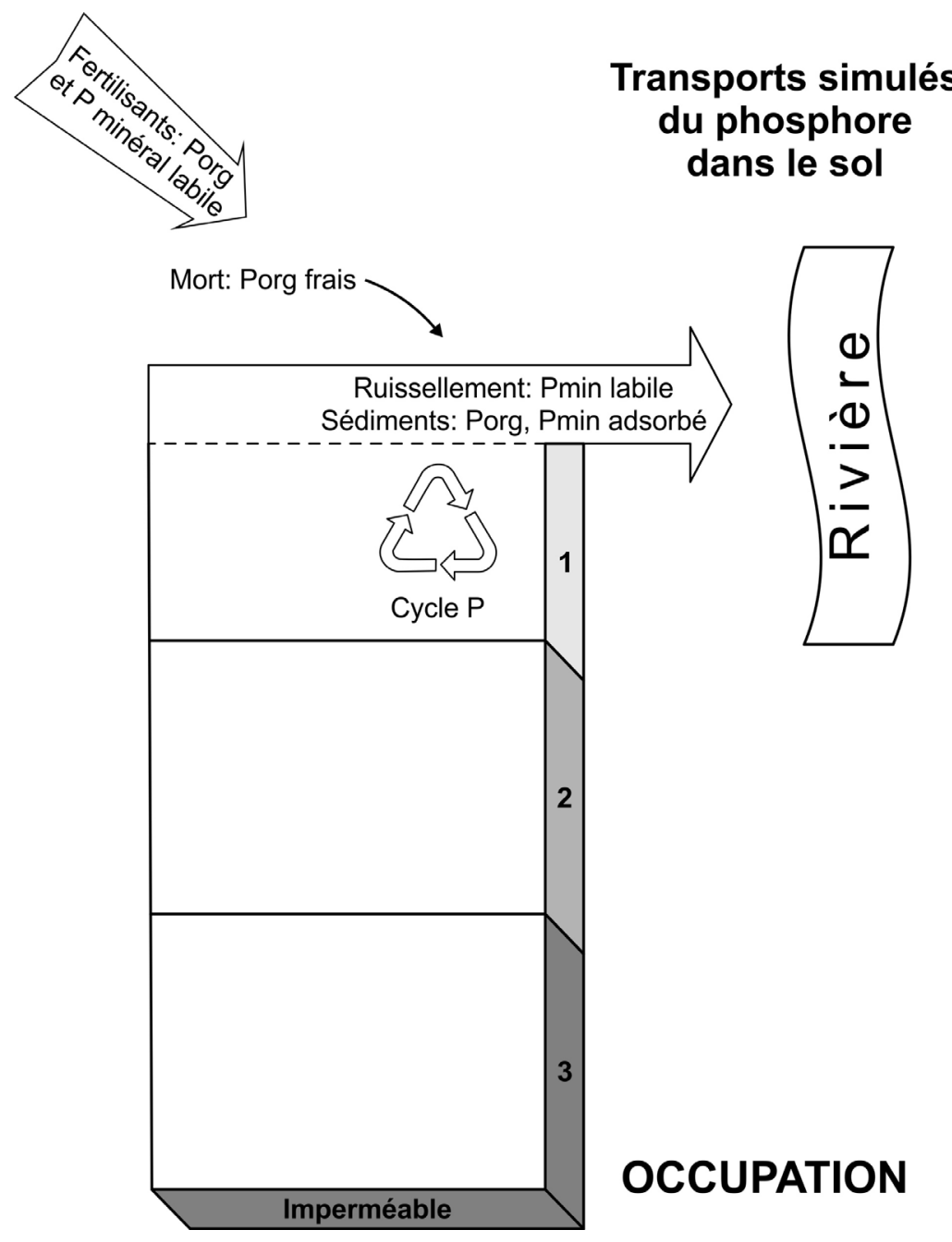

Figure 5. Transport simulé du phosphore dans le sol (VILLENEUVE et al., 1998). Simulated transport of phosphorus in soil (VILLENEUVE et al., 1998).

c) température et d) érosion et transport des sédiments. Les différentes variables simulées sur chaque tronçon du réseau hydrographique sont les suivantes : oxygène dissous (OD), demande biochimique en oxygène à cinq jours $\left(\mathrm{DBO}_{5}\right)$, algues (chlorophylle a), coliformes fécaux, phosphore organique $\left(\mathrm{P}_{\text {org }}\right)$, phosphore dissous $\left(\mathrm{P}_{\text {diss }}\right)$, azote organique $\left(\mathrm{N}_{\text {org }}\right)$, azote ammoniacal $\left(\mathrm{N}-\mathrm{NH}_{4}\right)$, nitrites $\left(\mathrm{N}-\mathrm{NO}_{2}\right)$ et nitrates $\left(\mathrm{N}-\mathrm{NO}_{3}\right)$.

La structure du modèle Qual-GIBSI est présentée à la figure 6. Les équations complètes de Qual-GIBSI sont inclues dans VILLENEUVE et al. (1998); quelques explications sont données ci-dessous.

L'oxygène dissous en rivière est une des principales variables utilisées afin de caractériser la qualité de l'eau et elle est la composante centrale des processus simulés par Qual-GIBSI. Les principales sources d'oxygène dissous sont la réaération atmosphérique, la production par photosynthèse et le flux entrant. L'oxygène dissous intervient dans les processus de la demande biochimique en oxygène, de l'oxydation des matières organiques, de la sédimentation et de la respiration des algues. La demande biochimique en oxygène est la quantité d'oxygène requise pour oxyder la matière carbonée dans le milieu. Les facteurs influençant la demande biochimique en oxygène sont la désoxygénation et la sédimentation. Dans Qual-GIBSI, la quantité de coliformes est utilisée comme indicateur de contamination microbiologique de l'eau de surface et la chlorophylle $a$ est utilisée pour estimer la concentration en biomasse des algues. La croissance des algues dépend de la disponibilité en lumière, en phosphore et en azote.

On distingue quatre formes d'azote dans le modèle : azote organique, ammoniac, nitrites et nitrates. La concentration des types d'azote dépend de la sédimentation, de la transformation d'un type d'azote à un autre, de la décomposition et de la consommation par les algues.

Le phosphore se présente sous deux formes dans le modèle : phosphore organique et phosphore dissous. Le phosphore organique provient de la décomposition des algues et est 


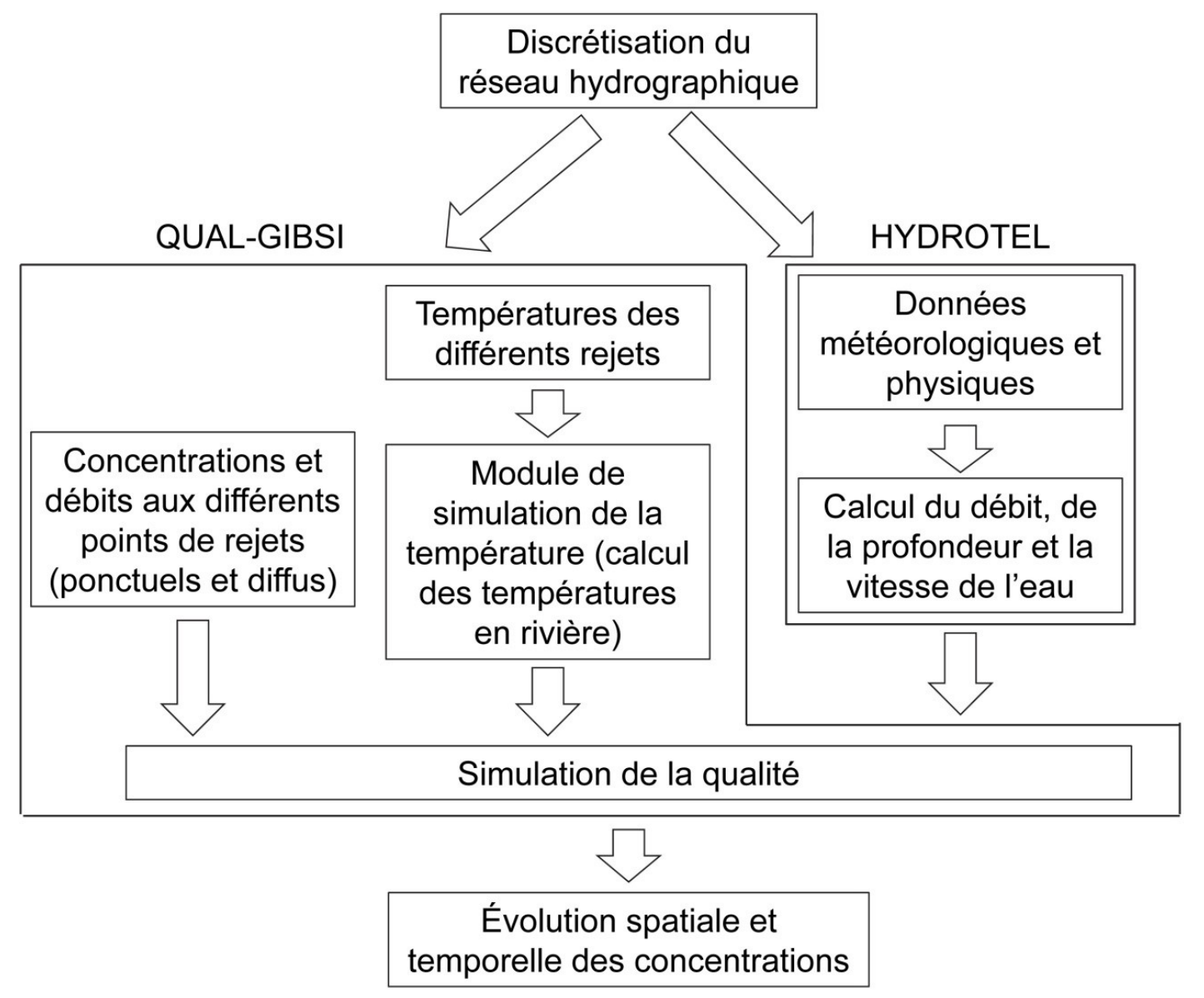

Figure 6. Structure globale de simulation du modèle Qual-GIBSI (adapté de VILLENEUVE et al., 1998).

Comprehensive simulation structure of the Qual-GIBSI model (adapted from VILLENEUVE et al., 1998).

transformé en phosphore dissous, qui est consommé par les algues.

\subsubsection{Calage}

Le calage du modèle est un procédé au cours duquel la valeur de certains paramètres est modifiée, au besoin, afin de représenter le mieux possible les données observées. Un calage par essais et erreurs est utilisé dans cette étude. Les modèles PO et Qual-GIBSI sont calés en même temps, sur la base d'observations de la qualité de l'eau en rivière. Le calage réalisé est de type global, c'est-à-dire qu'il prend en compte toute la région d'étude et affecte la même valeur, pour chaque paramètre, à tous les UHRH de la zone d'étude. Les variables de calage sont : la concentration en oxygène dissous (OD), la demande biochimique en oxygène à cinq jours $\left(\mathrm{DBO}_{5}\right)$, les algues (chlorophylle $a$ ), l'azote total, le phosphore total et les coliformes.

Les paramètres de calage de Qual-GIBSI sont sélectionnés à partir d'une analyse de sensibilité. Ce qui nous permet de considérer pour chaque variable de calage ses paramètres les plus influents. Les données de la qualité de l'eau ont été collectées de 2005 à 2007 par l'Agence de protection de l'environnement et de 2008 à 2009 par l'Académie des Sciences et des Technologies du Vietnam (ASTV). Le détail des données collectées est donné dans AUDET (2013). Les paramètres dont la valeur a été déterminée lors du calage sont ceux listés au tableau 1.

\subsubsection{Critère d'évaluation}

Pour évaluer la performance des simulations de qualité de l'eau, la NRMSE (normal root mean square error) est utilisée dans plusieurs études (LE, 2005; TAFTEH et al., 2013). Dans cette étude, on utilise la NRMSE comme indicateur d'évaluation de la performance des simulations.

$$
\text { NRMSE }=\frac{\sqrt{\frac{\sum_{i=1}^{n}\left(S_{i}-O_{i}\right)^{2}}{n}}}{\bar{O}}
$$

où $S_{i}$ est une valeur simulée, $O_{i}$ est une valeur observée, $\bar{O}$ est une valeur observée moyenne et $n$ est le nombre de données observées. 
Tableau 1. Valeurs des paramètres après calage global du modèle PO.

Table 1. Parameter values after global calibration of the PO model.

\begin{tabular}{|c|c|c|c|c|}
\hline Cycle & Processus & Paramètre & Description & Valeur calibrée \\
\hline \multirow[t]{5}{*}{ Azote } & $\begin{array}{l}\text { Transport des nitrates par } \\
\text { ruissellement }\end{array}$ & $\operatorname{limc}$ & $\begin{array}{l}\text { Limitation des quantités de } \\
\text { nitrates exportées }\end{array}$ & 0,15 \\
\hline & $\begin{array}{l}\text { Minéralisation entre l'azote } \\
\text { organique actif et les nitrates }\end{array}$ & $\mathrm{cmn}$ & $\begin{array}{l}\text { Constante de vitesse de l'humus } \\
(1 / \text { jour })\end{array}$ & 0,003 \\
\hline & \multirow[t]{2}{*}{ Dénitrification des nitrates } & $\operatorname{cdn}$ & Constante de dénitrification & -3 \\
\hline & & limdenit & $\begin{array}{l}\text { Facteur servant à limiter la } \\
\text { dénitrification }\end{array}$ & 0,5 \\
\hline & $\begin{array}{l}\text { Transport de l'azote par les } \\
\text { sédiments }\end{array}$ & $\mathrm{a} 0$ et $\mathrm{a} 1$ & $\begin{array}{l}\text { Paramètres dans le calcul du } \\
\text { facteur d'enrichissement }\end{array}$ & 0,75 et 1,05 \\
\hline \multirow[t]{4}{*}{ Phosphore } & $\begin{array}{l}\text { Minéralisation entre le } \\
\text { phosphore organique et le } \\
\text { phosphore dissous }\end{array}$ & $\mathrm{cmn}$ & $\begin{array}{l}\text { Constante de vitesse de l'humus } \\
(1 / \text { jour })\end{array}$ & 0,003 \\
\hline & $\begin{array}{l}\text { Transport du phosphore par } \\
\text { ruissellement }\end{array}$ & $\mathrm{dkd}$ & $\begin{array}{l}\text { Rapport entre la concentration } \\
\text { du phosphore labile dans les } \\
\text { sédiments et dans l'eau }\end{array}$ & 175 \\
\hline & $\begin{array}{l}\text { Transport de phosphore par les } \\
\text { sédiments }\end{array}$ & $\mathrm{a} 0$ et $\mathrm{a} 1$ & $\begin{array}{l}\text { Paramètres dans le calcul du } \\
\text { facteur d'enrichissement }\end{array}$ & 0,75 et 1,05 \\
\hline & & limsed & $\begin{array}{l}\text { Paramètre servant à diminuer la } \\
\text { quantité de phosphore disponible } \\
\text { à l'extraction par les sédiments }\end{array}$ & 0,33 \\
\hline
\end{tabular}

La NRMSE peut varier de 0 à l'infini. La valeur 0 indique que les résultats simulés reproduisent parfaitement les données observées. La valeur 1 signifie que l'erreur moyenne est égale à la moyenne des valeurs observées. Dans le cas où la NRMSE dépasse 1 , cela signifie que la moyenne des valeurs observées est meilleure comme prédicteur des valeurs observées que les résultats de simulation.

\section{APPLICATION AU BASSIN VERSANT DE LA RIVIẼRE CAU DU VIETNAM}

\subsection{Description de la zone d'étude}

\subsubsection{Situation géographique}

Situé au nord du Vietnam, le bassin versant de la rivière Cau a une superficie d'environ $6030 \mathrm{~km}^{2}$. Il traverse six provinces qui sont : Bac Kan, Thai Nguyen, Bac Giang, Bac Ninh, Ha Noi et Vinh Phuc (Figure 7). Dans le cadre de cette étude, la partie en amont de ce bassin versant constitue la région d'étude. Cette région comprend deux provinces, Bac Kan et Thai Nguyen, et couvre une superficie de $2760 \mathrm{~km}^{2}$, soit environ $45 \%$ de la superficie du bassin versant de la rivière Cau. La région d'étude s'étend de $21^{\circ} 20^{\prime}$ à $22^{\circ} 20^{\prime}$ de latitude Nord et de $105^{\circ} 28^{\prime} 1$ " à $106^{\circ} 8^{\prime} 1$ " de longitude Est. L'exutoire de la région d'étude est à la station Gia Bay (Figure 7) située à la ville de Thai Nguyen. Dans la région d'étude, les zones forestières se situent en amont tandis qu'en aval on retrouve une bonne densité de population et d'industries.

\subsubsection{Climat}

La région d'étude est située en zone tropicale soumise à la mousson avec deux saisons par année : une saison des pluies, de mai à octobre, caractérisée par un climat chaud et humide, et une saison sèche, de novembre à avril (plus fraîche). La vitesse du vent varie de 0,8 à $1,7 \mathrm{~m} \cdot \mathrm{s}^{-1}$. Les températures moyennes journalières varient de 10 à $35^{\circ} \mathrm{C}$ avec une température moyenne de $18{ }^{\circ} \mathrm{C}$ en saison sèche et de $27^{\circ} \mathrm{C}$ en saison des pluies. L'ensoleillement est de 1200 à 1800 h par année. L'évaporation annuelle varie de 750 à $1000 \mathrm{~mm}$. L'humidité relative moyenne varie entre 81 et $87 \%$. Les moyennes des précipitations varient de 1500 à $2700 \mathrm{~mm}$ par année, dont 80 à $90 \%$ sont enregistrées pendant la saison des pluies. Les précipitations mensuelles les plus importantes sont enregistrées aux mois de juillet et août, avec plus de $300 \mathrm{~mm}$, et les précipitations les plus faibles sont aux mois de décembre et janvier, avec environ $25 \mathrm{~mm}$. 


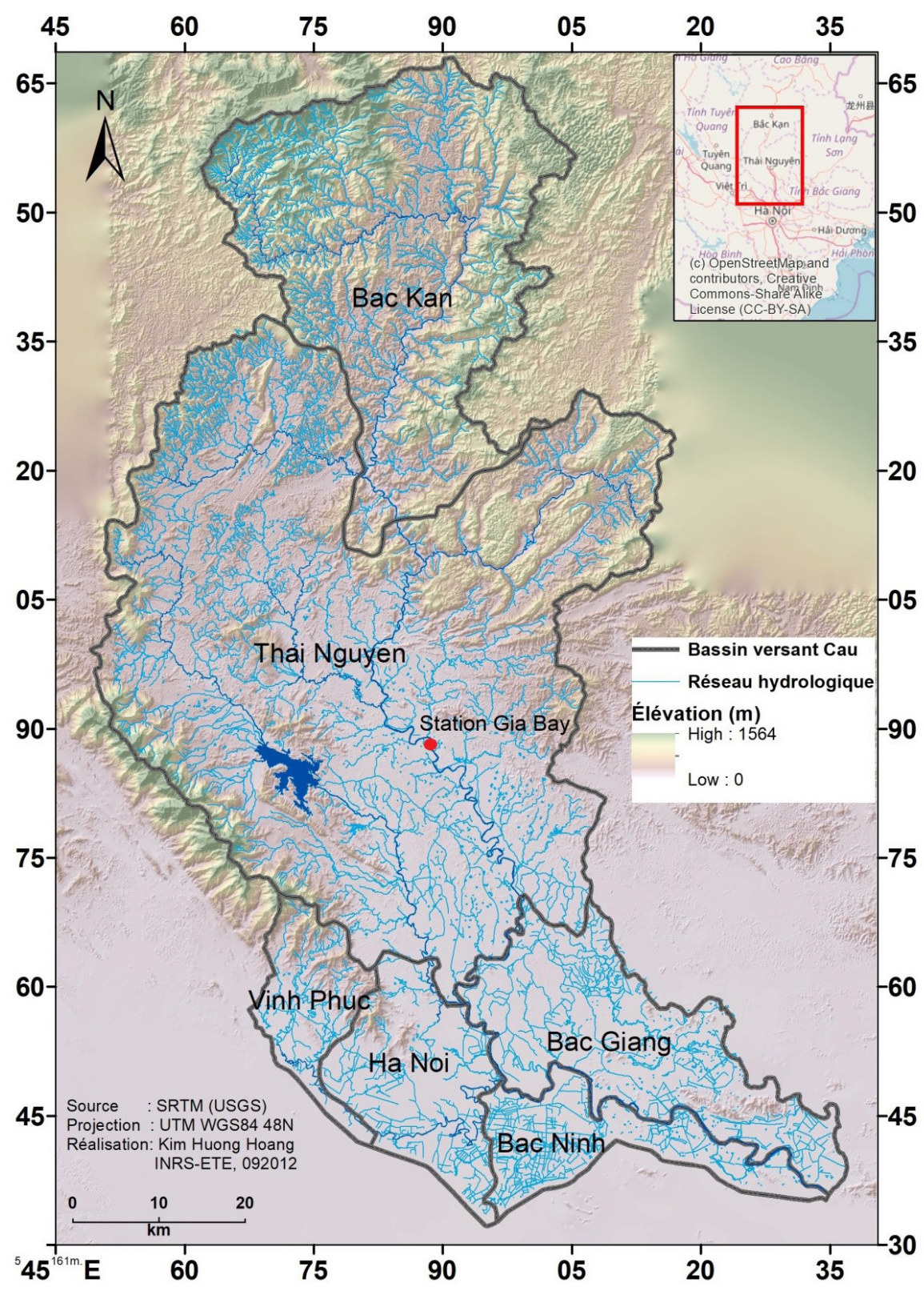

Figure 7. Bassin versant de la rivière Cau. Cau River watershed.

\subsubsection{Hydrologie}

$\mathrm{La}$ rivière Cau prend sa source au mont Van On, à $1175 \mathrm{~m}$ au nord de la province de Bac Kan. La rivière Cau est la principale rivière du bassin versant avec une longueur de $165 \mathrm{~km}$. Les principaux affluents de la rivière Cau sont les rivières Cho Chu (36,5 km de longueur), Nghinh Tuong (46 km), Du (44,5 km) et Cong $(96 \mathrm{~km})$.

Le volume d'eau de la saison des inondations représente de 75 à $85 \%$ du volume d'eau annuel.

\subsubsection{Population}

Selon une statistique réalisée au Vietnam en 2008 (BUREAU DE LA STATISTIQUE DU VIETNAM, 2008), le nombre d'habitants dans la région d'étude est d'environ 1,3 million avec un taux de croissance annuelle de 1,2\%. La densité moyenne de la population de la région d'étude est de 266 habitants. $\mathrm{km}^{-2}$. La plus faible densité est notée dans la commune de Bach Thong de la province Bac Kan (54 habitants $\cdot \mathrm{km}^{-2}$ ) et la plus forte densité est notée dans les provinces de Hanoi et Bac Ninh avec 2000 habitants $\cdot \mathrm{km}^{-2}$. Les eaux usées d'origine domestique sont jetées directement à l'environnement sans traitement. En milieu urbain, avec les systèmes de drainage, les eaux usées sont collectées et jetées à la rivière comme sources de pollution ponctuelle. En milieu rural, il n'existe pas de système de drainage; c'est pourquoi les eaux usées domestiques rurales sont considérées comme des sources de pollution diffuse. 


\subsubsection{Topographie et type de sol}

La topographie de la zone d'étude est complexe et est composée de montagnes, de vallées et de plaines. Le bassin versant a une pente allant du nord-ouest au sud-est. En amont, il y a des crêtes de montagnes qui excèdent les $1000 \mathrm{~m}$. Laltitude moyenne du bassin versant est de $190 \mathrm{~m}$.

La vitesse de l'eau est plus élevée en amont qu'en aval à cause du relief (la pente diminue d'amont en aval). On constate que la vitesse de l'eau à la station Thac Rieng est 1,5 fois plus grande que celles aux stations Thac Buoi et Gia Bay (IET, 2009). Par conséquent, le taux de sédimentation est faible en amont et élevé en aval.

En ce qui concerne le type de sol, la plaine du bassin versant est dominée par les alluvions alors que les sols argileux prédominent sur la montagne. Les montagnes rocheuses à la commune Vo Nhai correspondent au mont calcaire Thuong Nung, le plus grand de la province de Thai Nguyen.

\subsubsection{Utilisation du sol}

Selon la carte de l'utilisation du sol des deux provinces Bac Kan et Thai Nguyen du bassin versant de la rivière Cau (Figure 8), les terres agricoles occupent la plus grande proportion du territoire avec 50,1\%. Les zones forestières, quant à elles, occupent $41,1 \%$, alors que la classe urbaine n'occupe que $4,3 \%$ et les zones humides représentent 2,8 \%.

Les activités agricoles comprennent essentiellement l'élevage et la culture de plantes. Le cheptel de la région d'étude est composé principalement de bovins, de porcins et de volailles. Selon les données du département de statistique de l'année 2008 (BUREAU DE LA STATISTIQUE DU VIETNAM, 2008), le nombre de bovins est de 180000 contre 550000 porcins et 5500000 volailles. Les cultures du bassin versant sont les céréales (le riz pour 12600 ha et le maïs pour 82048 ha), les cultures fruitières et industrielles (35 376 ha) et d'autres plantes comme le thé et l'arachide, entre autres (22 $783 \mathrm{ha}$ ). Dans la pratique agricole, les fertilisants utilisés incluent les fumiers et lisiers des animaux et les engrais commerciaux. Les fertilisants non absorbés par les plantes constituent les sources de pollution diffuse agricole.

Les activités industrielles comprennent l'exploitation des mines, la production de papier, la production d'acier, etc. La plupart des activités industrielles sont concentrées à l'aval du bassin versant et les eaux qui y sont utilisées ne sont pas traitées et sont directement jetées à la rivière. Ces eaux usées sont considérées comme des sources de pollution ponctuelle.

\subsection{7 État de pollution de l'eau}

L'élevage a connu une croissance de 8,9\% par année de 2001 à 2006 (DAO, 2009). Une partie des rejets des animaux

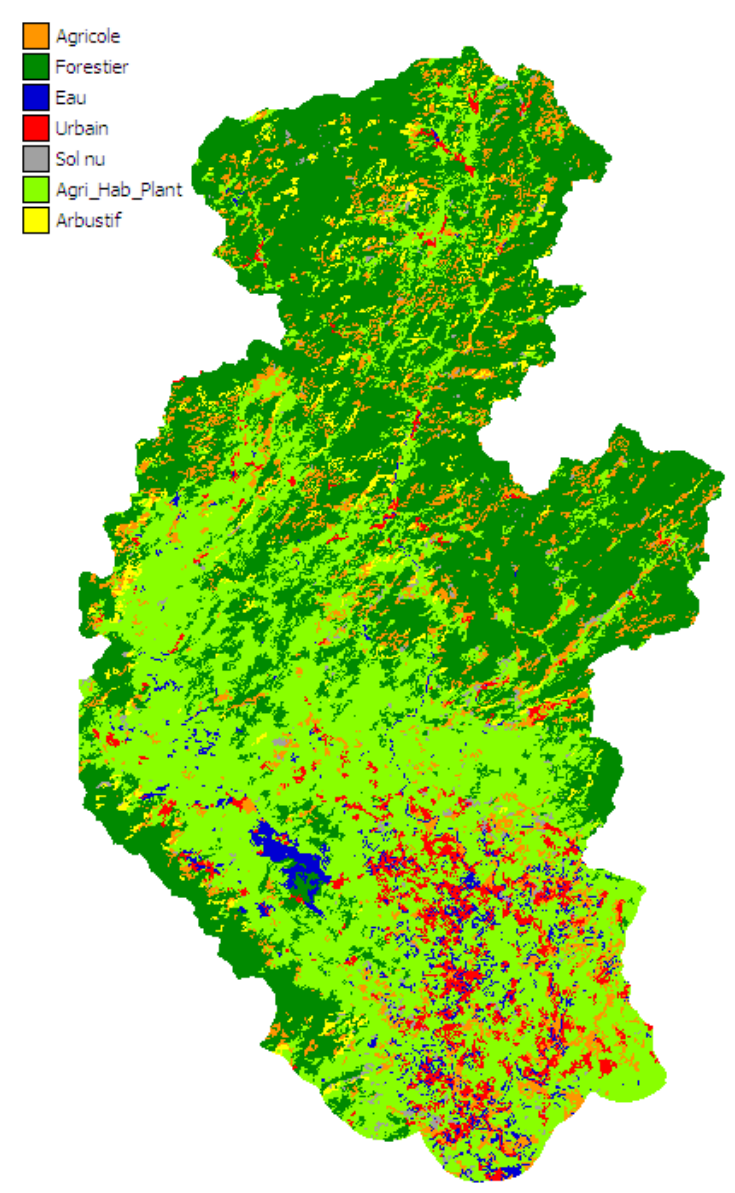

Figure 8. Utilisations du sol de la région d'étude (HOANG, 2007).

Land uses in the study area (HOANG 2007).

est utilisée par les agriculteurs comme fertilisants tandis que le reste est rejeté directement dans l'environnement. Cela constitue une très grande source de pollution des eaux de la rivière Cau. À chaque période de culture, en plus du fumier du cheptel, d'importantes quantités de fertilisants chimiques sont utilisées pour augmenter le rendement des cultures. En effet, pour une année, la quantité totale d'engrais chimique utilisée sur les surfaces agricoles dans ce bassin versant peut dépasser $3250 \mathrm{~kg} \cdot \mathrm{ha}^{-1}$. Le tiers de cette quantité d'engrais n'est pas absorbé par les plantes (MONRE, 2006). Ainsi, avec l'eau de ruissellement, plusieurs contaminants se retrouvent dans les cours d'eaux du bassin versant.

En 2006, le ministère des Ressources naturelles et de l'Environnement du Vietnam a déclaré que l'eau de la rivière Cau peut être considérée comme étant très polluée (MONRE, 2006). La figure 9 présente les valeurs moyennes annuelles de certaines variables de la qualité de l'eau $\left(\mathrm{OD}, \mathrm{DBO}_{5}, \mathrm{~N}-\mathrm{NH}_{4}\right.$ et $\mathrm{NO}_{3}$ ) en plusieurs points le long de la rivière Cau. Ces mesures ont été effectuées de l'amont vers l'aval. 

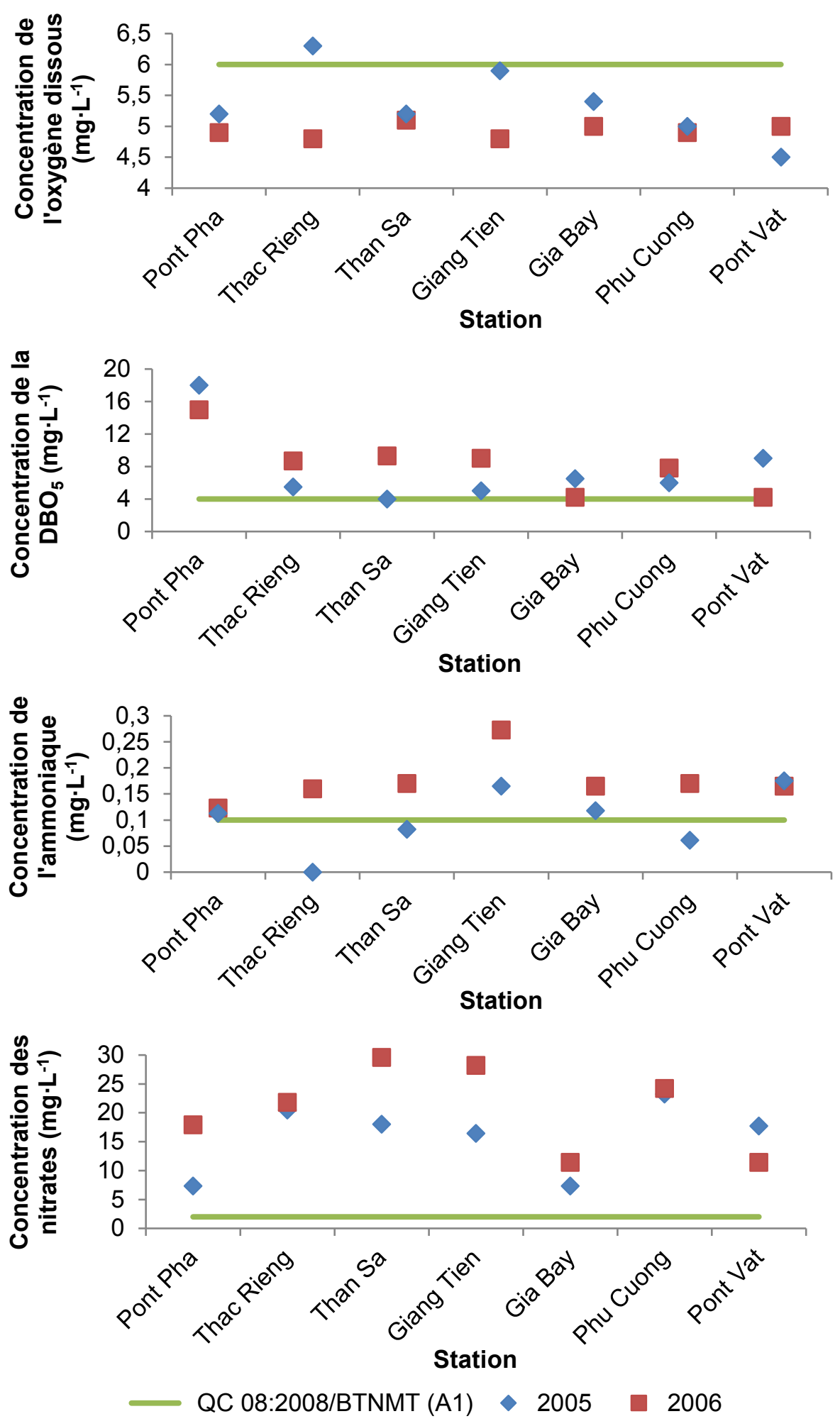

Figure 9. Concentrations mesurées des contaminants le long de la rivière Cau; la ligne horizontale verte correspond au critère de qualité de l'eau du Vietnam pour l'eau de surface (MONRE, 2005 et 2006)

Measured concentrations of contaminants along the Cau River; the green horizontal line corresponds to Vietnam's water quality criterion for surface water (MONRE, 2005 and 2006). 


\subsubsection{Sources de pollution}

Comme plusieurs autres bassins versants du Vietnam, le bassin versant de la rivière Cau fait face à de nombreuses utilisations abusives de l'eau par les populations, les activités agricoles et l'industrie. Ces activités humaines dans le bassin versant de la rivière Cau ont causé de sérieux problèmes de pollution qui entraînent une dégradation de la qualité de l'eau de la rivière. Le ministère de l'Environnement du Vietnam a répertorié les sources de pollution principales dans le bassin versant (MONRE, 2006).

\subsection{Résultats}

\subsubsection{Résultats du calage}

Les valeurs obtenues après calage global des paramètres des modèles PO et Qual-GIBSI sont données aux tableaux 1 et 2.

Pour analyser la qualité des simulations, les résultats de la station de Gia Bay (voir localisation sur la figure 7) sont utilisés. Cette station se trouve sur le cours principal de la rivière Cau et est celle qui possède le plus de données observées. La performance des résultats obtenus à la suite de la simulation des variables relatives à la qualité de l'eau a été évaluée en utilisant l'indicateur NRMSE. Pour chaque variable de qualité de l'eau, la NRMSE est calculée. Nous présentons dans ce qui suit les résultats de simulation des différentes variables de qualité de l'eau à la station de Gia Bay.

\subsubsection{Oxygène dissous}

La courbe des concentrations simulées et observées de l'oxygène dissous de la station de Gia Bay (l'exutoire de la région d'étude) est présentée à la figure 10 .

\subsubsection{Demande biochimique en oxygène à 5 jours}

Pour la $\mathrm{DBO}_{5}$, la courbe des concentrations simulées et observées de la station de Gia Bay des années 2008 et 2009 est présentée à la figure 11 .

\subsubsection{Chlorophylle $a$}

Les résultats de la simulation de la chlorophylle $a$ pour la station de Gia Bay des années 2008 et 2009 sont présentés à la figure 12 .

\subsubsection{Coliformes}

Pour les coliformes, la courbe des concentrations simulées et observées de la station de Gia Bay des années 2008 et 2009 est présentée à la figure 13 .

\subsubsection{Cycle du phosphore}

Les résultats pour le phosphore organique et dissous sont illustrés respectivement aux figures 14 et 15 .

\subsubsection{Cycle de l'azote}

Les résultats pour l'azote organique, l'azote ammoniacal et les nitrates sont illustrés respectivement aux figures 16,17 et 18.

Tableau 2. Valeurs des paramètres après calage global du modèle Qual-GIBSI.

Table 2. Parameter values after global calibration of the Qual-GIBSI model.

\begin{tabular}{llc}
\hline Processus & Paramètre et description & Valeur calée \\
\hline Oxygène dissous & $K_{2}:$ Taux de réaération & Option 2 \\
& $K_{4}:$ Demande des sédiments en oxygène $\left(\mathrm{g} \cdot \mathrm{m}^{-2} \cdot \mathrm{j}^{-1}\right)$ & 4 \\
Demande biochimique & $K_{1}:$ Taux de décroissance de la DBO $(\mathrm{l} / \mathrm{jour})$ & 0,4 \\
en oxygène à cinq & $K_{3}:$ Taux de décroissance de la DBO par déposition $(1 /$ jour $)$ & 0 \\
jours $\left(\mathrm{DBO}_{5}\right)$ & & \\
Coliformes & $K_{5}:$ Taux de décroissance des coliformes $(1 /$ jour $)$ & 2,8 \\
Chlorophylle $a$ & $\sigma_{1}:$ Taux de déposition des algues $\left(\mathrm{m} \cdot \mathrm{j}^{-1}\right)$ & 0,45 \\
& $\mu:$ Taux de croissance maximum des algues $(1 /$ jour $)$ & 1,2 \\
& $\rho:$ Taux de respiration des algues $(1 /$ jour $)$ & 0,12 \\
Cycle du phosphore & $\sigma_{2}:$ Source benthique de phosphore dissous $\left(\mathrm{mg} \mathrm{P} \cdot \mathrm{m}^{2} \cdot \mathrm{j}^{-1}\right)$ & -1 \\
& $\sigma_{5}:$ Coefficient de déposition du phosphore organique $(1 /$ jour $)$ & 0,01 \\
& $\beta_{4}:$ Coefficient de décroissance du phosphore organique $(1 /$ jour $)$ & 0,1 \\
& $\sigma_{3}:$ Source benthique pour l'azote ammoniacal $\left(\mathrm{mgN} \cdot \mathrm{m}^{2} \cdot \mathrm{j}^{-1}\right)$ & -1 \\
& $\sigma_{4}:$ Coefficient de déposition pour l'azote organique $(1 /$ jour $)$ & 0,01 \\
& $\beta_{1}:$ Coefficient pour l'oxydation de l'azote ammoniacal $(1 /$ jour $)$ & 0,5 \\
& $\beta_{2}:$ Coefficient pour l'oxydation des nitrites $(1 /$ jour $)$ & 1 \\
& $\beta_{3}:$ Coefficient pour l'hydrolyse de l'azote organique $(1 /$ jour $)$ & 0,1 \\
& $F:$ Coefficient de préférence pour l'azote ammoniacal des algues & 0,5 \\
\hline
\end{tabular}




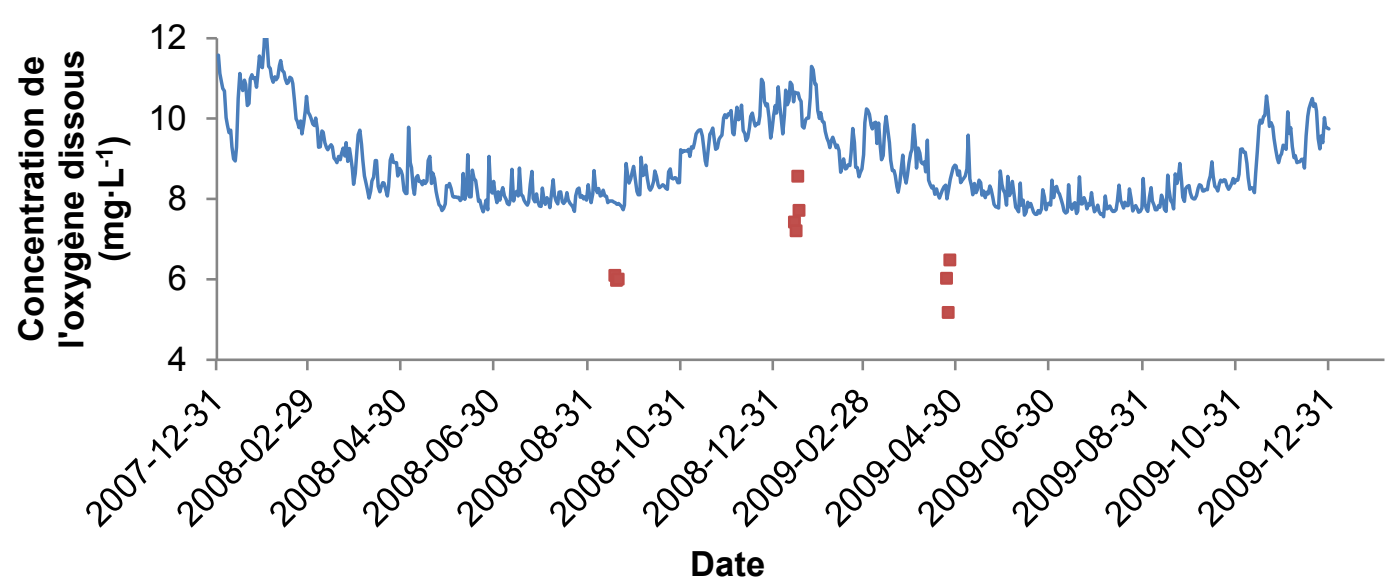

- Oxygene dissous observé —oxygene dissous simulé

Figure 10. Variations de l'oxygène dissous à la station aval Gia Bay de 2008 à 2009.

Dissolved oxygen variations at Gia Bay downstream station from 2008 to 2009.

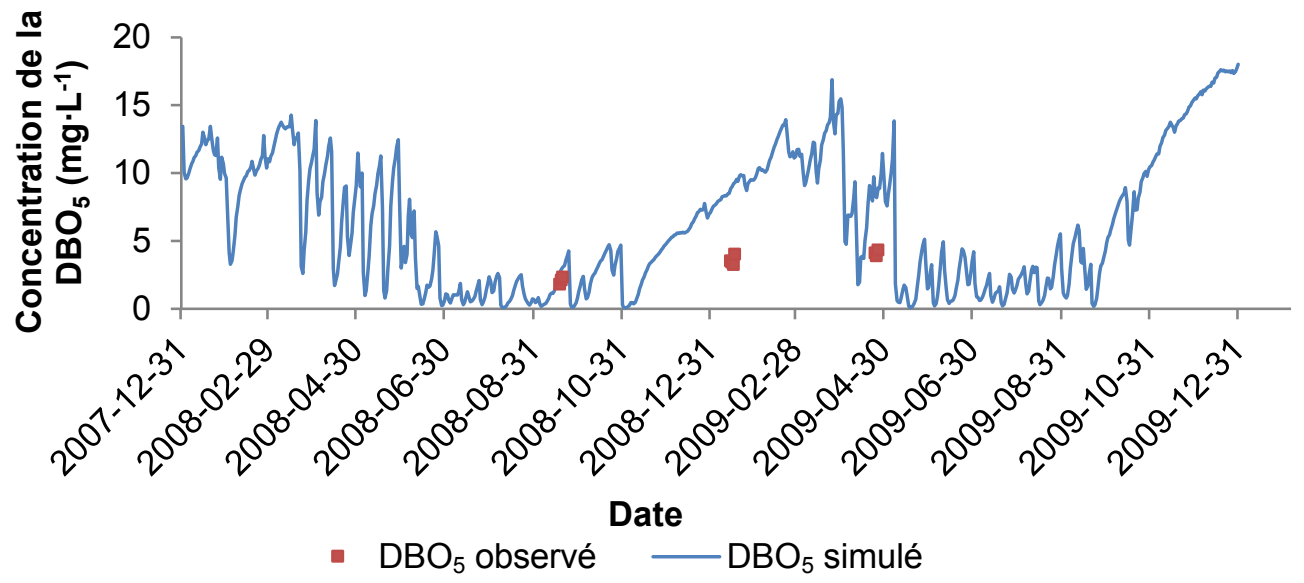

Figure 11. Variations de la demande biochimique en oxygène à cinq jours $\left(\mathrm{DBO}_{5}\right)$ à la station aval Gia Bay de 2008 à 2009.

Variations in biochemical oxygen demand at 5 days (BOD $)_{5}$ at Gia Bay downstream station from 2008 to 2009.

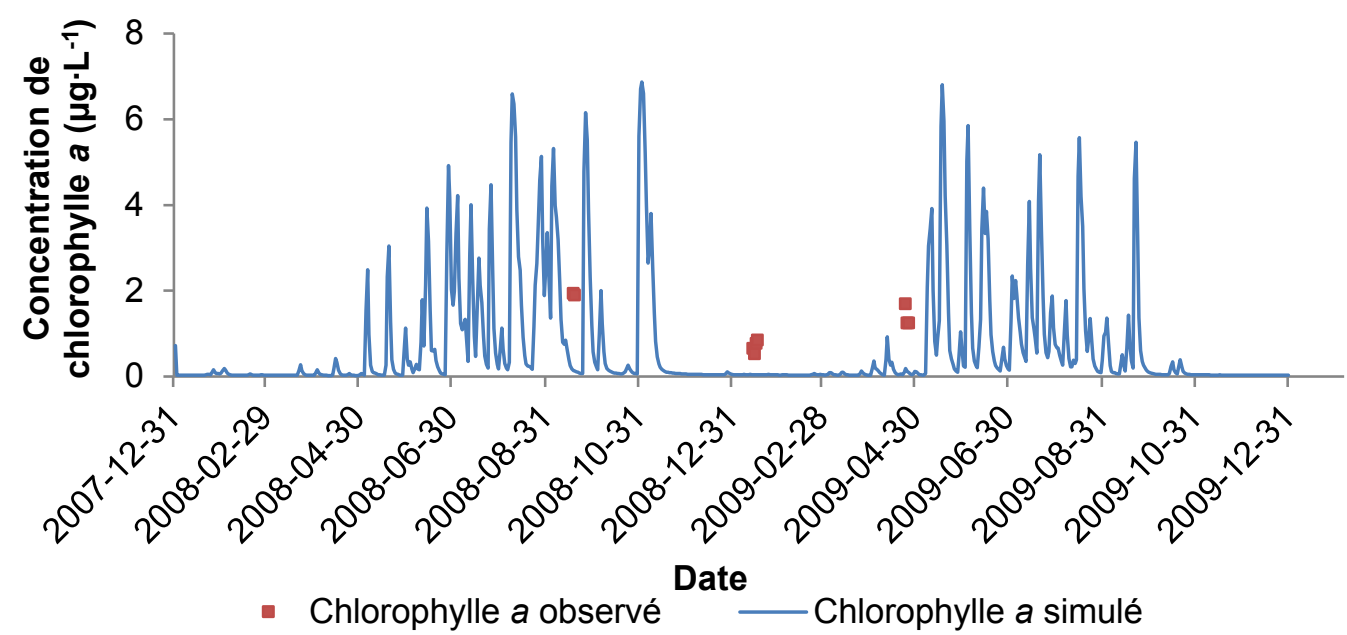

Figure 12. Variations de la chlorophylle $a$ à la station aval Gia Bay de 2008 à 2009. Chlorophyll a variations at Gia Bay downstream station from 2008 to 2009. 


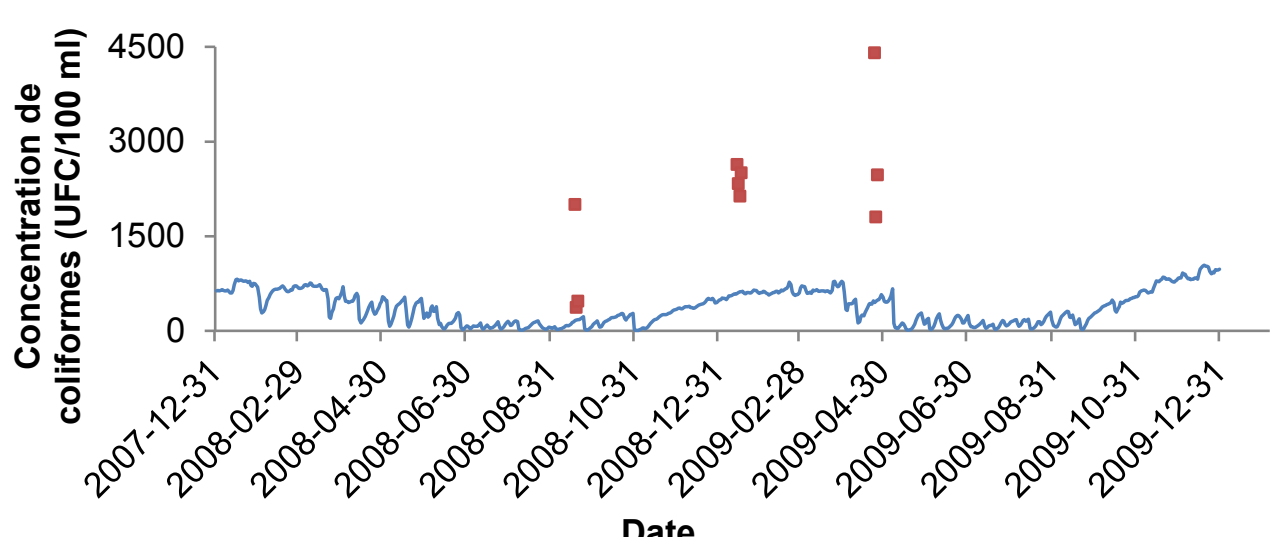

- Coliformes observés — Coliformes simulés

Figure 13 Variations des coliformes à la station aval Gia Bay de 2008 à 2009.

Coliform variations at Gia Bay downstream station from 2008 to 2009.

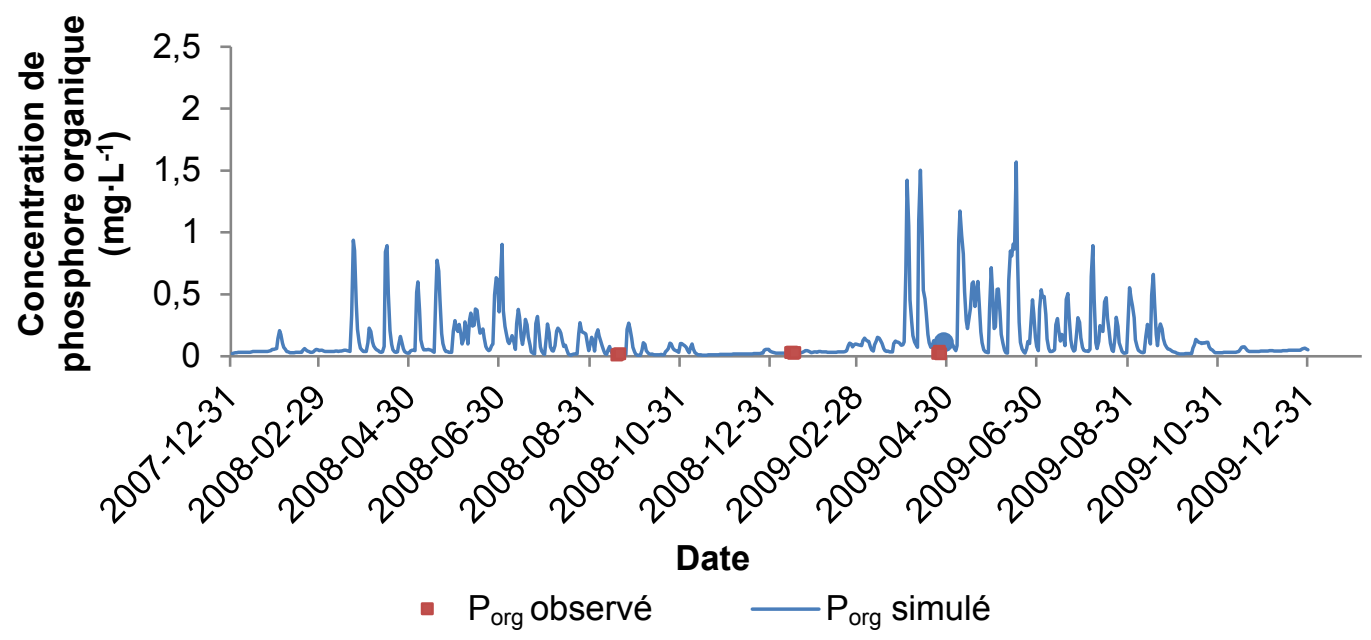

Figure 14 Variations du phosphore organique $\left(P_{\text {org }}\right)$ à la station aval Gia Bay de 2008 à 2009.

Organic phosphorus $\left(P_{\text {org }}\right)$ variations at Gia Bay downstream station from 2008 to 2009.

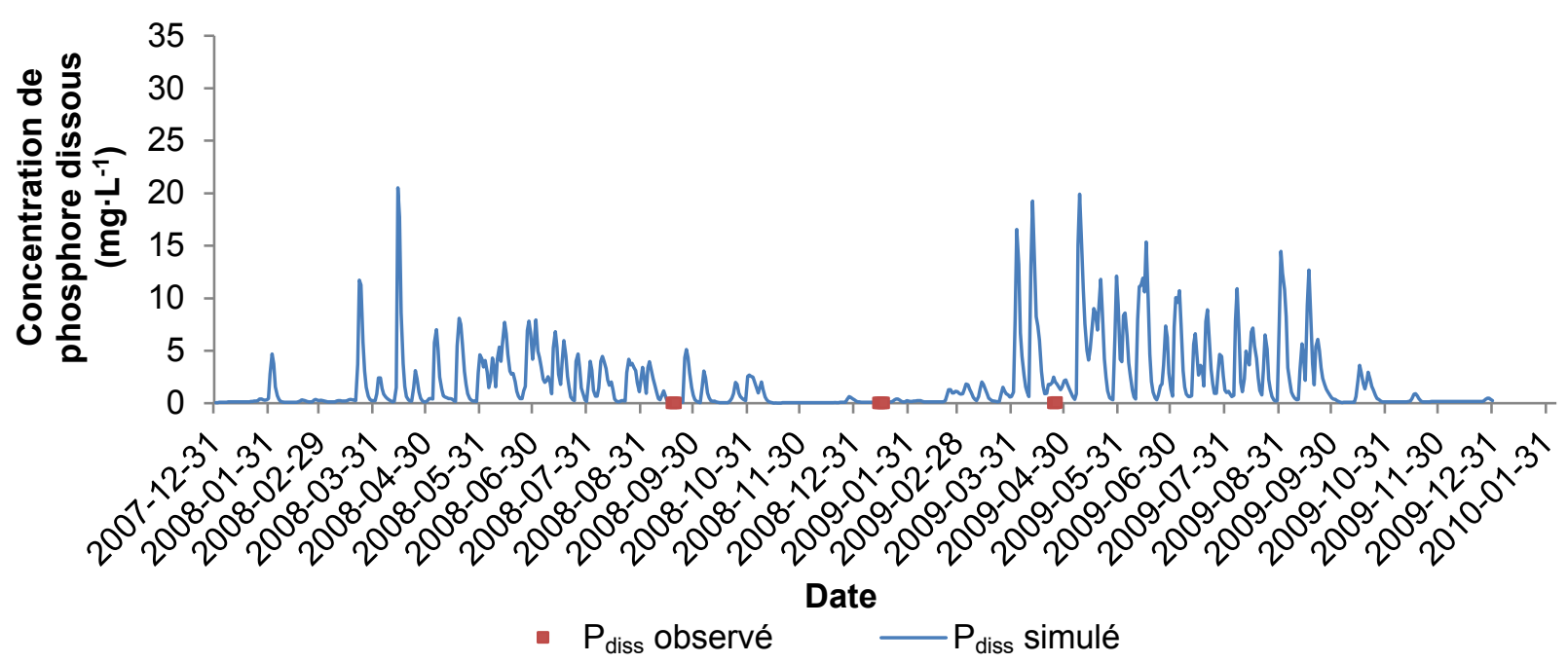

Figure 15 Variations du phosphore dissous $\left(P_{\text {diss }}\right)$ à la station aval Gia Bay de 2008 à 2009.

Dissolved phosphorus $\left(P_{\text {diss }}\right)$ variations at Gia Bay downstream station from 2008 to 2009. 


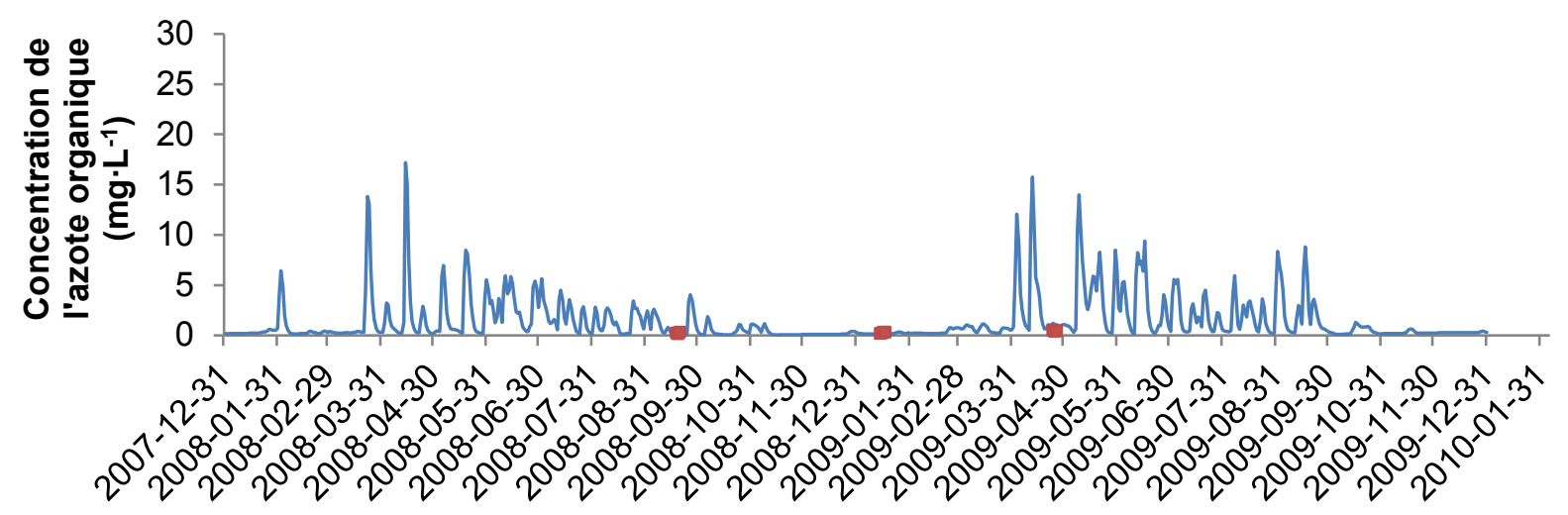

Date

- Norg observé

- Norg simulé

Figure 16 Variations de l'azote organique $\left(\mathrm{N}_{\mathrm{org}}\right)$ à la station aval Gia Bay de 2008 à 2009. .

Organic nitrogen $\left(N_{\text {org }}\right)$ variations at Gia Bay downstream station from 2008 to 2009.

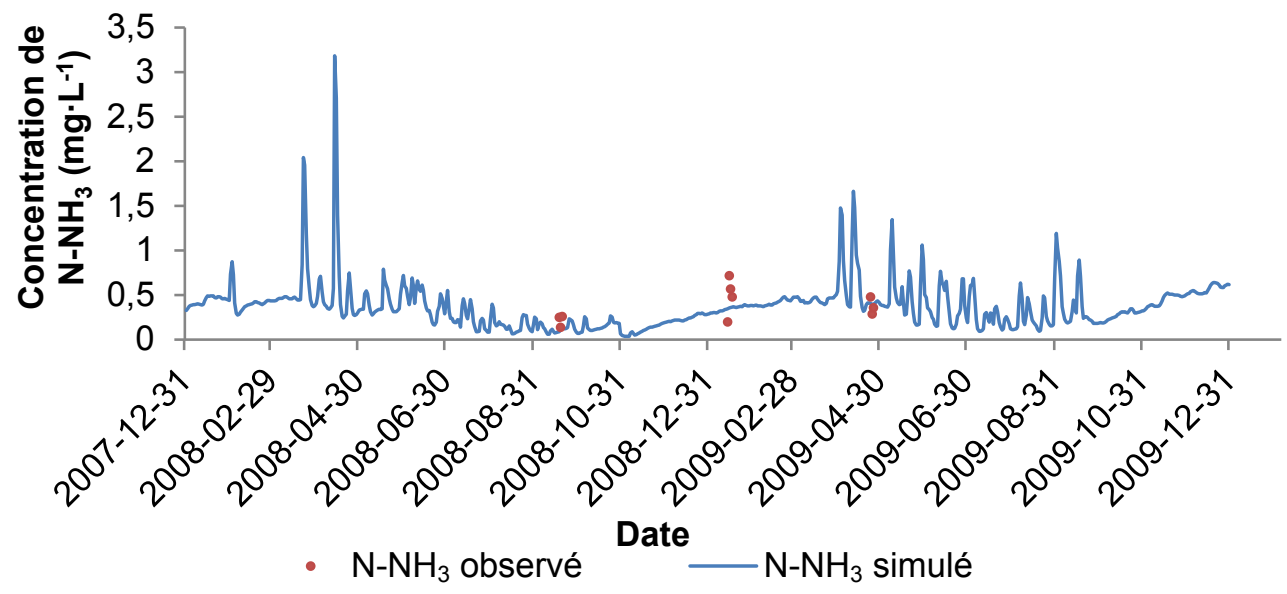

Figure 17 Variations de l'azote ammoniacal $\left(\mathrm{N}_{-} \mathrm{NH}_{3}\right)$ à la station aval Gia Bay de 2008 à 2009.

Ammonia nitrogen $\left(\mathrm{N}-\mathrm{NH}_{3}\right)$ variations at Gia Bay downstream station from 2008 to 2009.

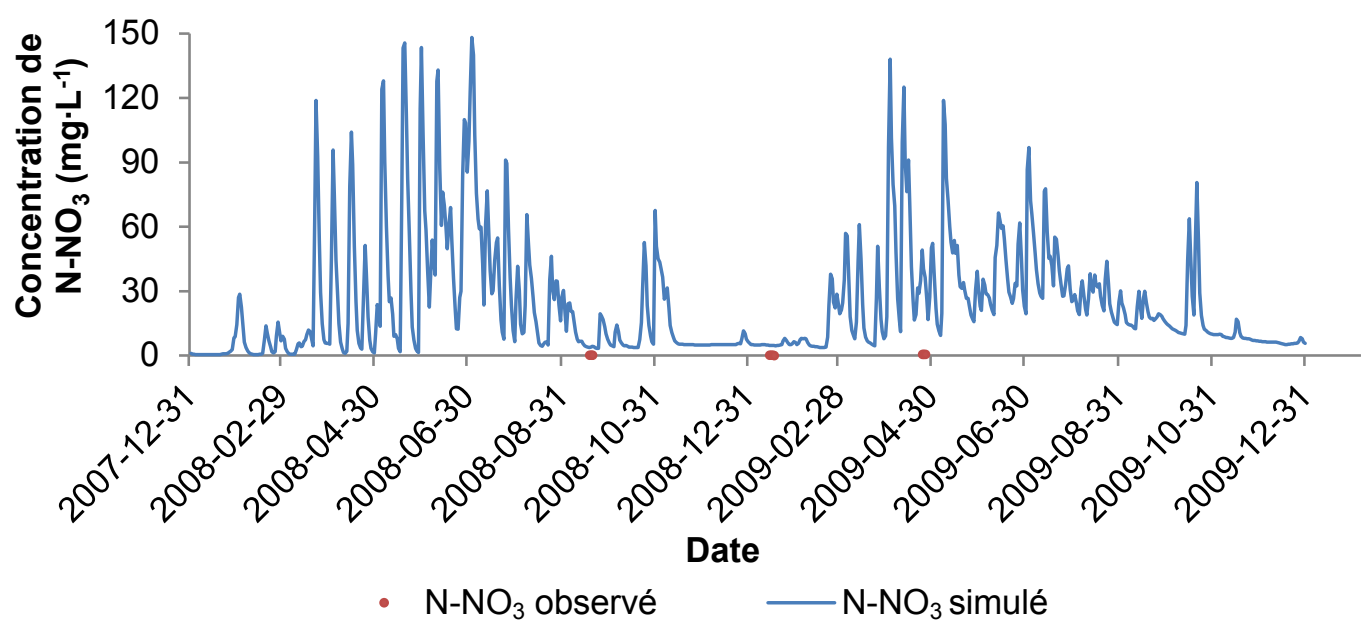

Figure 18 Variations des nitrates $\left(\mathrm{N}-\mathrm{NO}_{3}\right)$ à la station aval Gia Bay de 2008 à 2009.

Nitrate (N-NO $)_{3}$ variations at Gia Bay downstream station from 2008 to 2009. 


\subsubsection{Sommaire des résultats}

Les valeurs de l'indicateur de performance NRMSE pour les variables de qualité de l'eau pour les stations Thac Rieng, Cho Moi, Thac Buoi, Gia Tien et Gia Bay sont présentées dans le tableau 3.

Rappelons que les processus d'hydrologie, simulés par le modèle Hydrotel, sont à la base de la simulation de l'érosion et du transport des sédiments, du transport des nutriments et de l'évolution de la qualité de l'eau en rivière. Le modèle MODEROS simule les processus d'érosion et de transport de sédiments du bassin versant (PHAM, 2013). Or, le modèle Hydrotel a été calibré avec des données à une seule station, celle de Gia Bay, puisqu'on ne disposait pas de données ailleurs sur le bassin, ce qui peut entrainer des imprécisions dans les simulations plus en amont de la station. De plus, il faut préciser qu'il y a peu de données observées de qualité de l'eau et que ces données ont été prises en conditions de faibles débits. Par conséquent, des difficultés ont été notées lors du calage. Ainsi, les résultats de simulation des modèles PO et Qual-GIBSI pourraient être affectés par ces imprécisions.

Il est important de noter que malgré les imprécisions notées dans les résultats de simulation des modèles Hydrotel et MODEROSS ainsi que la rareté des données observées de qualité de l'eau, nous avons obtenu de bons résultats de simulation des variables de qualité de l'eau sauf pour l'OD et les coliformes.

\subsubsection{Vérification du respect du critère vietnamien de qualité de l'eau}

Afin de vérifier si les normes vietnamiennes de qualité de l'eau en rivière sont respectées, on évalue à l'aide des résultats de la modélisation la fréquence de dépassement de ces critères. À titre d'exemple, on a choisi le site de la station Gia Bay pour effectuer cette vérification. On aurait pu effectuer cette vérification en n'importe quel autre site sur la rivière puisque le modèle simule la qualité tout au long du cours d'eau.

Pour obtenir la fréquence de dépassement de la concentration d'une variable de qualité de l'eau par rapport au critère vietnamien de qualité de l'eau (CVQE), on calcule le nombre de fois (sur les 731 jours de simulation) où ce critère est dépassé ou mis à défaut (dans le cas où la concentration doit être inférieure au critère), puis on divise ce nombre par 731. Cette fréquence de dépassement est calculée pour chacune des variables de qualité de l'eau dont le CVQE a été défini au Vietnam par QCVN 08 : 2008/BTNMT (A1) et est résumée dans le tableau 4.

\subsubsection{Oxygène dissous}

La concentration en OD est inférieure au CVQE à la fin de la saison sèche à la station Gia Bay. La fréquence de dépassement de cette concentration y est de $9,3 \%$.
Tableau 3. Valeurs de NRMSE (normal root mean square error) à la station Gia Bay.

Table 3. Normal root mean square error (NRMSE) values at Gia Bay station.

\begin{tabular}{lc}
\hline Variable & NRMSE \\
\hline Oxygène dissous & 0,19 \\
DBO $_{5}$ & 0,68 \\
Coliformes & 0,67 \\
Chlorophylle $a$ & 0,35 \\
Azote organique & 0,80 \\
$\mathrm{NH}_{4}$ & 0,49 \\
$\mathrm{NO}_{3}$ & 0,72 \\
$\mathrm{Phosphore} \mathrm{organique}$ & 0,57 \\
Phosphore dissous & 0,92 \\
\hline
\end{tabular}

Tableau 4. Fréquence de dépassement du critère vitenamien de qualité de l'eau (CVQE).

Table 4. Frequency of exceeding the Vietnam's water quality criterion $(C V Q E)$.

\begin{tabular}{lcc}
\hline Variable & CVQE & $\begin{array}{c}\text { Fréquence de } \\
\text { dépassement (\%) }\end{array}$ \\
\hline Oxygène dissous & $>6 \mathrm{mg} \cdot \mathrm{L}^{-1}$ & 9,3 \\
$\mathrm{DBO}$ & $<4 \mathrm{mg} \cdot \mathrm{L}^{-1}$ & 48,4 \\
Coliformes & $<2500 \mathrm{UFC} / 100 \mathrm{ml}$ & 45,6 \\
Phosphore dissous & $<0,1 \mathrm{mg} \cdot \mathrm{L}^{-1}$ & 0 \\
$\mathrm{NH}_{4}$ & $<0,1 \mathrm{mg} \cdot \mathrm{L}^{-1}$ & 87,4 \\
$\mathrm{NO}_{3}$ & $<2 \mathrm{mg} \cdot \mathrm{L}^{-1}$ & 22,4 \\
\hline
\end{tabular}

4.2.2.2 Demande biochimique en oxygène à cinq jours

La concentration en $\mathrm{DBO}_{5}$, durant la période d'octobre à mars, est supérieure au CVQE à la station Gia Bay. La fréquence de dépassement du critère pour la $\mathrm{DBO}_{5}$ y est de $48,4 \%$.

\subsubsection{Coliformes}

La quantité de coliformes dans l'eau utilisée dans les activités agricoles durant la saison sèche dépasse le CVQE dans les villes de Bac Kan et Thai Nguyen. La fréquence de dépassement de la norme pour les coliformes est de 45,6\% pour la station Gia Bay.

\subsubsection{Phosphore dissous}

En ce qui concerne la concentration en phosphore dissous, sa fréquence de dépassement est de 0 pour les trois stations considérées, en particulier pour la station Gia Bay.

\subsubsection{Azote ammoniacal}

Quant à l'azote ammoniacal, on constate que la fréquence de dépassement da sa norme est la plus importante comparativement aux autres variables de qualité de l'eau. Elle est de $87,4 \%$ pour la station Gia Bay. 


\subsubsection{Azote-nitrate}

La concentration en azote-nitrate de l'eau, pendant la saison des pluies (station Gia Bay) ne respecte pas le CVQE. La fréquence de dépassement est de $22,4 \%$ pour la station Gia Bay.

\section{CONCLUSION}

Pour dresser un portrait de la qualité de l'eau dans la région à l'étude, les données de la qualité de l'eau collectées par MONRE de 2005 à 2006 ont été utilisées. Les données collectées lors de ces campagnes montrent que certaines variables de la qualité de l'eau de la rivière Cau ne respectent pas les critères concernant les eaux de surface. En effet, les valeurs des données associées à certaines variables, comme l'oxygène dissous, la demande biochimique en oxygène, l'azote ammoniacal et les nitrates, dépassaient souvent les critères en vigueur. Ceci démontre déjà que le niveau de pollution de la rivière Cau est élevé.

Dans cette étude, la qualité de l'eau en rivière a été simulée à l'aide de Qual-GIBSI pour la période de 2008 à 2009. D'abord, le modèle PO a été simulé pour déterminer la quantité d'azote et de phosphore en surface. Ensuite, en utilisant les résultats simulés par le modèle PO, le modèle Qual-GIBSI a été simulé.

Les résultats de simulation montrent que les variables de la qualité de l'eau, telles que l'oxygène dissous, la demande biochimique en oxygène, la chlorophylle $a$, les coliformes, l'azote organique, l'azote ammoniacal, les nitrates, le phosphore organique et le phosphore dissous, sont généralement bien simulées malgré la rareté de données qui caractérise la région d'étude. Ainsi, selon l'indicateur de performance utilisé (NRMSE), on peut considérer que les résultats de simulation du modèle sont acceptables. Nous avons également démontré dans cet article que, même lorsque les données observées sont rares, la modélisation permet d'évaluer la fréquence de dépassement de critères de qualité de l'eau.

La qualité de l'eau en rivière sur le bassin versant de la rivière Cau est influencée par les sources de pollution ponctuelle et diffuse. Durant la saison sèche, l'influence des rejets ponctuels sur la qualité de l'eau en rivière est plus importante que celle de la pollution diffuse, étant donné que le ruissellement y est très faible. Ainsi, la qualité de l'eau ne satisfait pas le critère vietnamien pour l'alimentation en eau municipale. Durant la saison des pluies, les concentrations d'OD, de $\mathrm{DBO}_{5}$ et de $\mathrm{NH}_{4}$ respectent le critère vietnamien, mais la pollution diffuse joue un rôle plus important en raison du transport de l'azote et du phosphore de surface par le ruissellement et les écoulements vertical et latéral. Cependant, la concentration du phosphore dissous a une fréquence de dépassement de $0 \%$ dans toutes les stations.

Rappelons que cette étude, dans ces applications, s'inscrit dans le cadre du projet "Gestion intégrée du bassin versant de la rivière Cau (Vietnam) ». Nous pensons que plusieurs aspects de ce projet pourraient être étoffés dans le futur afin d'en augmenter la portée.

Tout d'abord, la base de données contenant, entre autres, les caractéristiques du sol, l'occupation du sol, les informations relatives au cheptel et aux pratiques agricoles, et les données associées aux rejets et à la qualité de l'eau observée, devraient être complétées afin d'obtenir des résultats qui reflètent au mieux les réalités physiques du bassin versant.

De plus, l'insuffisance des données observées ne nous a pas permis de valider le calage du modèle comme on l'aurait souhaité dans le cadre de cette étude. Par conséquent, de nouvelles campagnes de collecte de données devraient être menées afin de permettre un meilleur calage et, surtout, une validation du modèle avec un jeu de données supplémentaires.

La pollution de l'eau par les pesticides a été identifiée dans le rapport de l'environnement du Vietnam de 2006 (MONRE, 2006) comme une préoccupation future du gouvernement vietnamien, mais jusqu'à maintenant, il n'existe pas d'étude portant sur ce sujet en ce qui concerne le bassin versant de la rivière Cau. Par conséquent, il serait intéressant de modéliser la pollution par les pesticides dans une étape subséquente du projet. Pour améliorer la qualité de l'eau du bassin versant, il faudrait également contrôler les sources de pollution. En ce qui concerne les sources de pollution ponctuelle, les plans de traitement des eaux usées devraient être mis en place afin que les eaux usées respectent les critères de qualité de l'eau existants (PHAM, 2013). Les sources de pollution diffuse d'origine agricole, autant en ce qui a trait au cheptel qu'en ce qui concerne l'utilisation d'engrais, constituent des apports importants d'azote et de phosphore en rivière. Il faudrait donc revoir les règles d'application des engrais de manière à mieux répondre aux besoins des plantes tout en réduisant la contamination qui découle de l'utilisation de ces fertilisants.

L'application du modèle GIBSI au bassin versant à l'étude montre que l'influence des sources de pollution sur la qualité de l'eau est très grande. Mais l'influence des rizières devrait également être prise en compte, par le biais du sous-modèle Hydrotel, étant donné que le stockage et le drainage de l'eau dans les rizières peuvent modifier le régime hydrologique et influencer les quantités de polluants dans la rivière. Les pratiques agricoles devraient par ailleurs être explorées en profondeur afin de mettre en place des mesures pour diminuer la pollution diffuse qu'elles peuvent occasionner. Le transport de pesticides est aussi un sujet à étudier. 
En terminant, soulignons que, sur la base des résultats présentés dans cet article, on peut conclure que les sousmodèles utilisés sont autant représentatifs des processus en climat tempéré qu'en climat tropical de type mousson. Ils ne demandent donc aucune modification dans leur formulation mathématique, sauf une attention particulière pour réaliser un processus de calage adapté, tel que celui présenté dans cet article.

\section{RÉFÉRENCES BIBLIOGRAPHIQUES}

AUDET L. (2013). Modélisation de la qualité de l'eau de la rivière Cau au Viet Nam. Mémoire de maîtrise, Univ. Québec, INRS, Canada, 198 p.

BEASLEY D.B. et L.F. HUGGINS (1981). ANSWER: Areal nonpoint source watershed environment response simulation. User's manual. United States Environmental Protection Agency Region V, EPA 905/9-82-001, Chicago (IL), ÉtatsUnis, $57 \mathrm{p}$.

BROWN L.C. et T.O. BARNWELL (1987). The enhanced stream water quality models, QUAL2E and QUAL2E UNCAS - Documentation and User's manual. United States Environmental Protection Agency, EPA/600/3-87/007, Athens (GA), États-Unis, 204 p.

BUREAU DE LA STATISTIQUE DU VIETNAM (2008). Niên giám thống kê các tỉnh Bắc Kạn, Thái Nguyên năm 2008. Annuaire statistique du Vietnam, des provinces de Bac Kan et Thai Nguyen. Hanoi, Vietnam.

DAO L.H. (2009). Thưc trạng và định huoóng bảo vệ môi trường trong chăn nuôi (Situation et orientation de la protection de l'environnement face à l'élevage). Ministère des ressources naturelles et de l'environnement du Vietnam (MONRE), Hanoi, Vietnam.

DUCHEMIN M., M. LACHANCE, G MORIN et R. LAGACÉ (2001). Approche géomatique pour simuler l'érosion hydrique et le transport des sédiments à l'échelle des petits bassins versants. Water Qual. Res. J. Can., 36, 435-473.

FORTIN J.P., R. MOUSSA, C. BOCQUILLON et J.P. VILLENEUVE (1995). Hydrotel, un modèle hydrologique distribué pouvant bénéficier des données fournies par la télédétection et les systèmes d'information géographique. Rev. Sci. Eau, 8, 97-124.
HOANG K.H. (2007). Les changements de l'occupation du sol et ses impacts sur les eaux de surface du bassin versant: Le cas du bassin versant de la rivière Câu (Viêt-nam). Mémoire de maîtrise, Univ. Québec, INRS, Canada, 127 p.

INSTITUTE OF ENVIRONMENTAL TECHNOLOGY (IET) (2009). Report on water quality monitering campaign for Cau River - Integrated Cau River basin management project. IET, Vietnam.

KNISEL W.G. (1980). CREAMS: A field-scale model for chemical, runoff, and erosion from agricultural management systems. United States Department of Agriculture, Conservation Research Report 26, Washington (DC), États-Unis, 672 p.

LEONARD R.A., W.G. KNISEL et D.A. STILL (1987). GLEAMS: Groundwater loading effects of agricultural management systems. Trans. ASAE, 30, 1403-1418.

LE B.T. (2005). An application of soil and water analysis tool (SWAT) for water quality of Upper Cong Watershed, Vietnam. Mémoire de maîtrise, Asian Institute of Technology (AIT), Thaillande, $110 \mathrm{p}$.

MINISTÈRE DES RESSOURCES NATURELLES ET DE L'ENVIRONNEMENT DU VIETNAM (MONRE) (2005). Báo cáo hiện trạng môi trường quốc gia năm 2005 (Rapport de l'environnement du Vietnam en 2005). MONRE, Hanoi, Vietnam.

MINISTÈRE DES RESSOURCES NATURELLES ET DE L'ENVIRONNEMENT DU VIETNAM (MONRE) (2006). Báo cáo hiện trạng môi truờng quốc gia năm 2006 (Rapport de l'environnement du Vietnam en 2006). MONRE, Hanoi, Vietnam.

NASR S. (1996). Modélisation du transport des nutriments et des pesticides à l'échelle d'un bassin versant. Rapport R-462, INRS, Centre Eau Terre Environnement, Québec (QC), Canada, 46 p.

NEITSCH S.L., J.G. ARNOLD, J.R. KINIRY et J.R. WILLIAMS (2005). Soil and water assessment tool Theoretical documentation, version 2005. Texas Agricultural Expriment Station, Blackand Research Center, Texas (TX), États-Unis, $494 \mathrm{p}$.

NEITSCH S.L., J.G. ARNOLD et J.R. WILIAMS (2011). Soil and water assessment tool - Theoretical documentation, version 2009. Texas Water Resources Institute, College Station (TX), États-Unis, 618 p. 
NGUYEN H.T. (2012). Apport de la modélisation hydrologique distribuée à la gestion intégrée par bassin versant des ressources en eau. Thèse de doctorat, Univ. Québec, INRS, Canada, $200 \mathrm{p}$.

ONGLEY E.D. (1996). Control of water pollution from agriculture. Food and Agriculture Organization of the United Nations (FAO), Rome, Italie, 101 p.

PHAM T.N. (2013). Modélisation de l'érosion et du transport sédimentaire sur les bassins versants soumis à un régime de mousson dans un contexte de gestion intégrée des ressources en eau. Thèse de doctorat, Univ. Québec, INRS, Canada, $278 \mathrm{p}$.

PLUARG (1979). International reference group on Great Lakes pollution from land use activities (PLUARG). http://agrienvarchive.ca/pluarg/pluarg.html (consultation le 28 septembre 2018).

QUILBÉ R. et A.N. ROUSSEAU (2007). GIBSI: an integrated modelling system for watershed management - sample applications and current developments. Hydrol. Earth Syst. Sci., 11, 1785-1795.

REFSGAARD J.C. et E. HANSEN (1982). A distributed groundwater/surface water model for the Susa catchment - Part 1: Model description. Nord. Hydrol., 13, 299-310.

ROUSSEAU A.N., A. MAILHOT, R. TURCOTTE, M. DUCHEMIN, C. BLANCHETTE, M. ROUX, N. ETONG, J. DUPONT et J.P. VILLENEUVE (2000). GIBSI - An integrated modelling system prototype for river basin management. Hydrobiologia, 422/423, 465-475.
SHARPLEY A.N. et J.R. WILLIAMS (1990). Epic erosion/ productivity impact calculator, model documentation. United States Department of Agriculture, Technical Bulletin Number 1768, Washington (DC), États-Unis, 377 p.

SHIKLOMANOV I.A. (1998). World water resources - A new appraisal and assessment for the $21^{\text {st }}$ century - A summary of the monograph world water resources. United Nations Educational, Scientific and Cultural Organization, Paris, France, $37 \mathrm{p}$.

TAFTEH A., H. BABAZADEH, N.A. EBRAHIMIPAK et F. KAVEH (2013). Evaluation and improvement of crop production functions for simulation winter wheat yields with two types of yield response factors. J. Agr. Sci., 5, 111122.

VILLENEUVE J.P., C. BLANCHETTE, M. DUCHEMIN, J.F. GAGNON, A. MAILHOT, A.N. ROUSSEAU, M. ROUX, J.F. TREMBLAY et R. TURCOTTE (1998). Rapport final du projet GIBSI : Gestion de l'eau des bassins versants à l'aide d'un système informatisé - Tome I. INRS, Centre Eau Terre Environnement, Québec (QC), Canada, 488 p.

YOUNG R.A., C.A. ONSTAD, D. BOSCH et W.P. ANDERSON (1987). AGNPS, agricultural non-pointsource pollution model - A watersheds analysis tool. United States Department of Agricultural, Conservation Research Report 35, Washington (DC), États-Unis, 80 p. 